\title{
Autopolyploid establishment depends on life history strategy and the mating outcomes of clonal architecture
}

1. Department of Biology, University of Toronto Mississauga, Mississauga, ON, L5L 1C6, Canada

2. Biology Department, Queen's University, Kingston K7L 3N6, Canada.

* author for correspondence: we.vandrunen@gmail.com

KEYWORDS: perenniality, life history, clonal reproduction, polyploid establishment, geitonogamy, unreduced gametes

RUNNING TITLE: Perenniality and clonality promote polyploidy

MAIN TEXT WORD COUNT: 7981

\section{LIST OF ELEMENTS:}

Main text -6 figures 


\section{ABSTRACT}

36 Polyploidy is a significant component in the evolution of many taxa, particularly plant groups.

37 However, the mechanisms promoting or preventing initial polyploid establishment in natural

38 populations are often unclear. We develop spatially explicit agent-based simulation models to

39 explore how perennial life history and clonal propagation influence the early stages of polyploid

40 establishment. Our models show that polyploid establishment is unlikely among short-lived

41 plants. Polyploids have increased establishment probability when both diploid and polyploid

42 lifespans are long, especially when unreduced gamete production is non-zero. Further, polyploids

43 that combine sexual and clonal reproduction can establish across a wide range of life history

44 strategies. Polyploid genets containing many, far spreading ramets are most successful, but

45 genets with tightly clumped ramets have similar establishment probability when pollen dispersal

46 is local and rates of self-fertilization are high. Clonal architecture has a substantial impact on the

47 spatial structure of the mixed cytotype population during establishment; altering patterns of

48 mating within or between cytotypes, the mechanisms through which polyploid establishment

49 proceeds, and the final composition of the polyploid population after successful establishment.

50 Overall, our findings provide insight into the complex relationship between polyploidy,

51 perenniality, and clonal reproduction, and offer testable predictions for future empirical work. 


\section{INTRODUCTION}

56 Polyploidy has played a key role in the evolutionary history and diversification of plants (Wood

57 et al. 2009; Husband et al. 2013), and there is growing evidence that it is also common within

58 many animal clades (Otto and Whitton 2000). Polyploids arise through whole-genome

59 duplication (WGD) events resulting in more than two complete chromosome sets in an

60 individual, which may occur through the hybridization of two species (allopolyploidy) or within

61 a single species (autopolyploidy). Because a WGD event can result in instant reproductive

62 isolation and drastic phenotypic change between a polyploid and its progenitors (Baack et al.

63 2015), polyploidy is often viewed as one of the quintessential examples of sympatric speciation.

64 Indeed, approximately $15 \%$ and $30 \%$ of speciation events in angiosperms and ferns respectively

65 have been attributed to polyploidization (Wood et al. 2009). Though polyploidy is prevalent

66 throughout plants and the wider tree of life, many of the ecological and evolutionary mechanisms

67 leading to the establishment success or failure of newly formed polyploids in natural populations

68 are poorly understood (Husband 2000; Ramsey and Ramsey 2014; Soltis et al. 2016b).

69 The rate of polyploid formation surely exceeds the rate of successful establishment (Ramsey

70 and Schemske 2002), because rare polyploids experience significant fitness disadvantages when

71 they first arise in populations dominated by their lower-ploid parents (Husband 2000; Ramsey

72 and Schemske 2002). Here, the majority of the polyploid mating opportunities are intercytotype,

73 resulting in inviable odd-ploidy offspring and low fitness, and ultimately polyploid exclusion

74 from the population (Levin 1975). This process of Minority Cytotype Exclusion (MCE) is

75 expected to be a significant barrier to polyploid evolution (Thompson and Lumaret 1992;

76 Husband 2000; Fowler and Levin 2016), and in many ways is analogous to the challenges faced

77 by a mutant genotype invading a resident population with a lack of compatible mates, or as 
78 individuals colonize new habitat (Baack 2005; Pannell et al. 2015). In general, polyploid

79 establishment is more probable when the effects of MCE are mitigated (Stebbins 1950), which

80 may occur through: 1) a shift in the balance of intra- vs. intercytotype mating, 2) a reduced

81 reliance on sexual reproduction, 3) high rates of polyploid formation, or 4) increased persistence

82 time.

83 The process of polyploid establishment and MCE are challenging to study empirically (e.g.,

84 Husband 2000; Sutherland et al. 2020), but previous theoretical treatments largely confirm that

85 polyploid establishment is facilitated by factors contributing to the four MCE-reducing processes

86 listed above. Studies have identified important roles for self-fertilization (Levin 1975; Rodríguez

87 1996; Rausch and Morgan 2005), local dispersal (Baack 2005), asexuality (Nakayama et al.

88 2002; Yamauchi et al. 2004; Spoelhof et al. 2020), elevated unreduced gamete (UG) production

89 (Felber and Bever 1997; Burton and Husband 2001; Husband 2004), and iteroparity (Rodríguez

90 1996). Most of these studies have explicitly represented plant systems and autopolyploidy (but

91 see Fowler and Levin 2016), but even within this framework there remain significant gaps in our

92 knowledge of the critical early period immediately following polyploid formation (Spoelhof et

93 al. 2017).

94 A perennial life history strategy and asexual reproduction through clonal propagation have

95 long been hypothesized to increase polyploid establishment success (Gustafsson 1948; Stebbins

96 1950; Rodríguez 1996; Chrtek et al. 2017), and both have the potential to reduce MCE via one or

97 more mechanisms. Perenniality (coupled with iteroparity) increases the persistence of a new

98 polyploid over several generations and the number of reproductive bouts during an individual's

99 lifetime, and should increase the likelihood of viable intracytotype mating via self-fertilization

100 (Gustafsson 1948; Otto and Whitton 2000; Rice et al. 2019). Additionally, perenniality and 
101 longer polyploid lifespans may influence the probability that neopolyploid individuals

102 originating from different WGD events will overlap in time, increasing opportunities for

103 successful polyploid outcrossing.

104 Unlike perenniality, clonal reproduction directly contributes to polyploid fitness through the 105 production of clonal offspring. Clonal propagation decreases the probability of complete

106 polyploid exclusion, and increases the probability of intracytotype mating through

107 geitonogamous self-fertilization between shoots within a genetic clone (i.e., a genet, comprised

108 of numerous genetically-identical ramets; Vallejo-Marín et al. 2010). Since mating in plants

109 generally occurs between near neighbours, the placement and arrangement of ramets can

110 dramatically alter the sexual fitness of clonal plants by affecting pollen transport within and

111 between genets (Handel 1985; Vandepitte et al. 2013; Van Drunen et al. 2015). The potential for

112 geitonogamy to benefit an establishing polyploid is then inherently dependent on both population

113 spatial structure and clonal architecture. Dense genets with closely packed ramets (i.e., phalanx

114 architecture; Charpentier 2002; Vallejo-Marín et al. 2010) exhibit high rates of geitonogamous

115 self-fertilization and little outcrossing. If the costs of inbreeding are low (Husband et al. 2008), a

116 polyploid with a phalanx strategy can create its own local pocket of same-cytotype mates (Li et

117 al. 2004; Baack 2005). In contrast, sparse genets with widely dispersed ramets (i.e., guerrilla

118 architecture Charpentier 2002) should promote intermingling between different genets and

119 mating via outcrossing. A guerrilla architecture and a high capacity for lateral growth could

120 promote the spread of polyploids throughout a population (Van Drunen et al. 2015; Herben et al.

121 2017), and may be a better strategy if inbreeding depression is high. Thus, though either type of

122 clonal architecture could promote polyploid establishment, the pathway to establishment could

123 be fundamentally different between strategies. However, only a handful of polyploid 
124 establishment models include spatial or population structure (Li et al. 2004; Baack 2005;

125 Spoelhof et al. 2020; Griswold 2021), and none have considered the effects of geitonogamy or 126 clonal architecture.

127 Evaluating the influence that perenniality or clonality have on polyploid establishment is 128 complicated by the correlations amongst these traits. At a broad scale, phylogenetic and 129 biogeographical studies have demonstrated a positive evolutionary association between 130 polyploidy and perenniality or clonality (Gustafsson 1948; Herben et al. 2017; Rice et al. 2019; 131 Van Drunen and Husband 2019), but these relationships may be confounded by the fact that all 132 clonal plants are perennial, and that the perennating organs of many plants are themselves clonal 133 modules (e.g., tillering grasses; Klimeš et al. 1997). Thus, the relative importance of perenniality 134 and clonality, and their potential interactions, during the initial stages of polyploid evolution are 135 not well-defined.

136 Successful polyploid establishment generally requires some difference between cytotypes 137 that conveys a benefit to the polyploids (Levin 1975), an idea corroborated by classic two138 species coexistence theory (reviewed in Barabás et al. 2018). Many models incorporate this 139 through niche shifts reducing competition between cytotypes (e.g., Rodríguez 1996), or by 140 directly setting polyploid fitness higher than that of diploids (e.g., Baack 2005). Relatively few 141 explicitly explore other phenotypic differences between cytotypes (but see Rausch and Morgan 142 2005; Chrtek et al. 2017; Griswold 2021), though in practice WGD can result in sweeping 143 changes to gene expression (Levin 2002; Soltis et al. 2016a), physiology (Maherali et al. 2009; 144 Anneberg and Segraves 2020), or morphology in new polyploids (Husband et al. 2016). WGD is 145 expected to produce bigger cells with long cell cycles, which should slow development, delay 146 maturity, and potentially increase lifespan (Bennett 1972; Levin 2002; Beaulieu et al. 2008; 
147 Blomme et al. 2014), resulting in polyploids that are more perennial than diploids. The

148 immediate effects of WGD on clonal reproduction are less predictable. Newly synthesized

149 polyploids can be more or less clonal than diploids (Van Drunen and Husband 2018a, 2018b;

150 Walczyk and Hersch-Green 2019), and established polyploids also show a similarly variable

151 relationship with clonality (e.g., Hroudová and Zákravský 1993; Keeler 2004; Baldwin and

152 Husband 2013). In any case, we may hypothesize that an increase in clonal reproduction will aid

153 polyploid establishment, while a decrease will almost certainly ensure failure for a polyploid

154 among highly clonal diploids.

155 Theory is a valuable tool in exploring the elusive early steps in polyploid evolution, and for

156 understanding how the traits implicated in these high-level patterns might causally influence key

157 individual- and population-level processes. Here, we explore the effects of perenniality and

158 clonal reproduction on the early stages of autopolyploid establishment using spatially explicit,

159 agent-based simulation models incorporating the complex dynamics between reproductive

160 strategy, perenniality, and population spatial structure. Specifically, we ask: 1) Does a perennial

161 life history increase polyploid establishment? 2) Does clonal reproduction increase polyploid

162 establishment? 3) How is polyploid establishment influenced by genet size and architecture? and

163 4) Do differences in life history or reproductive strategy between polyploids and diploids further

164 influence these processes?

\section{METHODS}

167 MODEL OVERVIEW:

168 Models were written in R v3.6.2 (R Development Core Team 2019), the full code is available

169 online (https://github.com/wevandrunen/autopolyploid-establishment-lifehistory- 
170 clonalarchitecture). Simulations take place on a square population lattice measuring D x D units.

171 The population lattice exhibits periodic boundary conditions, such that pollen, seed, or clonal

172 ramets that disperse beyond the grid limits in one direction re-enter the population on the

173 opposite edge (i.e., the grid is toroidal). Individuals occupy all cells in the lattice. and population

174 size $(\mathrm{N}=\mathrm{D} \times \mathrm{D})$ is constant over each generation. Models are initialized with $\mathrm{N}-1$ diploid

175 individuals, and a single tetraploid individual at a random location. The population then

176 undergoes a series of biological processes over a set number of time-steps, equivalent to growing

177 seasons or generations. For each generation, the sequence of events is: sexual and clonal

178 reproduction, survival, and offspring recruitment (fig. 1).

179 We make several assumptions about the equivalency of diploid vs. tetraploid individuals.

180 Cytotypes are equal competitors and occupy the same ecological niche, and diploids and

181 tetraploids have equal seed germination and ramet establishment probability. Finally, individual

182 fecundity and survival are not age related; individuals reproduce sexually and asexually

183 immediately upon recruitment into the population, have a fixed reproductive output per

184 generation, and have a constant probability of survival to the next generation over their lifetime.

STEP 1 - REPRODUCTION:

187 The first step in each generation is pollen dispersal and ovule fertilization (fig. 1). All individuals

188 are hermaphroditic and produce $o v$ ovules per generation (fig. 1). Pollen production is unlimited

189 and no ovule is left unfertilized, but not all fertilizations will result in viable seed (see below).

190 Ovule fertilization proceeds by first determining the pollen density from each individual in the

191 population at the location of the ovule source, including pollen produced by the ovule source

192 itself. Pollen dispersal is described by Gamma distribution $P(x)$ (eq. [1]) with shape parameter $\beta$ 
193 set to 1, corresponding to leptokurtic pollen distribution (fig. S1A; figs. S1-S12 and table S1 are

194 available online) where pollen density is highest at the pollen source $(x=0)$.

$$
P(x), S(x)=\alpha^{\beta} x^{\beta-1} \frac{e^{-\alpha x}}{\Gamma(\beta)}
$$

The rate parameter $\alpha$ is calculated as $\beta / d_{p}$, where $d_{p}$ is the average pollen dispersal distance

197 (fig. 1). Due to the toroidal shape of the population grid, pollen from an individual could arrive at

198 a location through multiple routes, and we calculate pollen densities using the shortest distance

199 between the pollen donor and ovule source. The selected pollen donor for each ovule is chosen

200 by the weighted pollen probability density at the ovule location. All pollen is assumed to be

201 equally able to fertilize an ovule: there is no pollen precedence, female mate choice, or

202 performance differences between reduced (1n) and unreduced ( $2 \mathrm{n})$ pollen.

The outcome of each mating event, and seed viability, is determined by the identity of the

204 gamete sources. A mating event may result in one of 22 cross-types, that fall into four categories

205 per cytotype: within shoot self-fertilization (autogamy), between ramet self-fertilization

206 (geitonogamy), intercytotype outcrossing, and intracytotype outcrossing (table S1). Rates of self-

207 fertilization vs. outcrossing are therefore emergent properties of the model, determined

208 intrinsically through pollen donor selection. Self-fertilized seeds are inviable with probability $k_{2}$

209 or $k_{4}$ and viable with probability $\left(1-k_{2}\right)$ or $\left(1-k_{4}\right)$, for diploids and tetraploids respectively

210 (table S1, fig. 1), which may be interpreted as early acting inbreeding depression. Diploids

211 produce unreduced gametes (2n) with probability $u g$, and reduced gametes (1n) with probability

$212(1-u g)$ (table S1, fig. 1). Tetraploids make only reduced gametes (2n), and the formation of

213 ploidy levels higher than tetraploid cannot occur. Intercytotype crosses, or intracytotype crosses

214 involving unreduced gametes, are viable only when the offspring is tetraploid, and triploidy is

215 assumed to be lethal (table S1). All seeds produced via intracytotype outcrossing with reduced 
216 gametes are viable. After fertilization, all viable seeds are stored in a common pool for

217 recruitment into the next generation.

218 Individuals can produce a number of clonal ramets in each generation, $c_{2}$ for diploids, and $c_{4}$

219 for tetraploids (fig. 1). The $c_{2}$ and $c_{4}$ parameters are constant throughout a single model run,

220 though they may differ from each other. For some scenarios, $c_{2}$ and/or $c_{4}$ are zero, meaning no

221 ramets are produced by individuals of that cytotype and reproduction is entirely sexual. Ramets

222 are stored in the same offspring pool as the seeds for the recruitment step.

224 STEP 2 - SURVIVAL:

225 Diploid and tetraploid individuals within the population survive between generations with

226 probability $s v_{2}$ or $s v_{4}$ (fig. 1). For annual life histories these probabilities are zero. For perennials

227 an average of $s v_{2}$ diploid and $s v_{4}$ tetraploid individuals survive between generations, and the

228 remainder of the population, up to $\mathrm{N}$, is supplemented by new recruits from the available

229 offspring pool.

231 STEP 3 - RECRUITMENT:

232 Offspring are recruited onto the sites newly vacated by deceased individuals, and may be either

233 sexually produced seedlings or clonal ramets. The recruitment process is similar to ovule

234 fertilization, where the probability of a recruit colonizing a vacant site is calculated as the seed or

235 ramet density at the site, dispersed from their source locations. Seed probabilities follow the

236 Gamma function $S(x)$ (eq. [1]), where $\beta$ is set to 1 and the rate parameter $\alpha$ is $\beta / d_{s}$, where $d_{s}$ is

237 the average seed dispersal distance (fig. 1). In contrast, ramet dispersal $C(x)$ is assumed to

238 conform to a bell-shaped Gaussian distribution (eq. [2], variance $\sigma^{2}=0.15$; fig. S1B) such that 
peak probability density occurs at the mean ramet dispersal distance from the parental source

$240 \quad\left(d_{C 2}\right.$ or $d_{C 4}$, fig. 1).

$$
C(x)=\frac{e^{-\left(x-d_{c}\right)^{2} /\left(2 \sigma^{3}\right)}}{\sqrt{2 \pi \sigma^{2}}}
$$

Recruitment sites are filled in random order, and offspring are removed from the common

243 pool following successful recruitment. Since ramet probability density is formulated to be high

244 relative to seed density at $x \approx d_{c}$ from a source plant (fig. S1), clonal recruitment will occur much

245 more frequently at these distances than seed colonization, mirroring a biologically realistic

246 scenario where the competitive and establishment ability of ramets is higher than that of

247 seedlings (Vallejo-Marín et al. 2010). Centering ramet colonization at $d_{c}$ results in higher ramet

248 density at a "spacer distance" away from the primary shoot and imposes strong spatial structure

249 on clonal genets (e.g., Winkler and Stöcklin 2002). After recruitment, seedlings are assigned new

250 unique genet IDs, while ramets retain the ID of their parent. Data on the survivors and new

251 cohort of individuals is passed to the next generation, and the reproduction step begins again.

PARAMETERIZATION:

254 There are 15 adjustable parameters in the model (fig. 1). Except where noted, simulations take

255 place on a square lattice with dimension $D=30$ and a population of $N=900$, which remains

256 constant across generations. Simulations begin with only one polyploid individual $(t=1)$. The

257 number of ovules produced per individual $(o v)$ is five for both diploids and tetraploids, resulting

258 in 4500 seeds per generation for a population of $N=900$. Under the most restrictive

259 circumstances for sexual reproduction (i.e., high selfed-seed inviability, limited pollen dispersal,

260 no unreduced gamete production, and similar cytotype frequencies), trials showed that at least

2612000 viable seeds were produced per generation; enough to minimize the possibility of 
anomalous long-distance dispersal events. Diploid individuals may produce unreduced gametes

263 (UGs) at frequency $u g$, and reduced gametes at frequency $1-u g$ (table S1).

Two parameters affect the realized amount of self-fertilization: pollen dispersal distance $\left(d_{p}\right)$

265 alters the probability of self-pollination (autogamous or geitonogamous), and selfed-seed

266 inviability $(k)$ alters the probability that self-fertilized offspring are included in the pool of viable

267 recruits (fig. 1, table S1). We focus on these two parameters because they are expected to be

268 influential on sexual fitness in clonal plants (Eckert 2000; Van Drunen et al. 2015), where

269 dispersal distance determines pollen kernel density (fig. S1), and regulates whether pollen is

270 likely to remain within a ramet, encounter other ramets in the same genet, or encounter ramets

271 from different genets. We test the effects of nine combinations of $d_{p}$ and $k(3 \times 3)$ across different

272 model scenarios, hereafter referred to as the core dispersal-inviability parameter set. The three

273 core value for average pollen dispersal distance $\left(d_{p}\right)$ were 0.5 grid units (pollen rarely exits the

274 source cell), 2 (local dispersal), and 5 (far dispersal) (figs. 1, S1). For simplicity, average seed

275 dispersal distances were assumed to mirror those of pollen $\left(d_{p}=d_{s}\right)$, and are equal between

276 cytotypes for all simulations. The probability of selfed-seed inviability for diploids $\left(k_{2}\right)$ and

277 tetraploids $\left(k_{4}\right)$ took the values $0.1,0.5$, or 0.9 (fig. 1) and were equal in most scenarios, where

278 larger values indicate stronger inviability or inbreeding depression.

279 The remaining six parameters differentiate the life history and reproductive strategies of

280 diploid or tetraploid individuals: per generation survival ( $s v_{2}$ and $s v_{4}$ ), clonal ramet production

$281\left(c_{2}\right.$ and $\left.c_{4}\right)$, and average ramet dispersal distance $\left(d_{C 2}\right.$ and $\left.d_{C 4}\right)$ (fig. 1). These parameters are

282 described in more detail in the following sections. 
We first examine the effect of perenniality on polyploid establishment in the absence of clonal

286 reproduction $\left(c_{2}=c_{4}=0\right)$. Diploid and tetraploid individuals may have the same probability of

287 survival between generations $\left(s v_{2}=s v_{4}\right)$, or survival probabilities may differ $\left(s v_{2} \neq s v_{4}\right)$. For

288 equal strategies, $s v_{2}$ and $s v_{4}$ ranged from 0 (a short-lived annual strategy with zero survival

289 probability between generations) to 0.9 (a long-lived perennial strategy with a $90 \%$ survival

290 probability) in increments of 0.1 (fig. 1). For the core dispersal-inviability parameter set, there

291 were 90 unique scenarios where diploids and polyploids have equal life-histories.

Because genome duplication is expected to slow growth rates and increase lifespans, we

293 only consider tetraploid survival probability exceeding that of diploids, defining a "Tetraploid

294 Survival Advantage" as $s v_{4}-s v_{2}$. Diploid survival probability per generation ranged from 0 to

2950.8 , while tetraploid survival could take any larger value (e.g., for $s v_{2}=0, s v_{4}$ can be $0.1-0.9$ ).

296 This results in 45 pairs of $s v_{2}$ and $s v_{4}$, and a total of 405 parameter combinations.

We ran several additional simulations to determine how alternate model formulations could influence the effect of life history on tetraploid establishment. For these models, we consider:

1) Low population density - Imposing a fully occupied grid may misrepresent the population

300 structure of some plants; more closely resembling a population of long-lived trees than patchy

301 herbaceous species. Less local competition for recruitment sites may increase polyploid

302 establishment potential. We reran the full set of 495 life history simulations on a larger

303 population lattice of $D=40$ units with population size constant at $N=900$ randomly distributed

304 individuals, for a $\sim 45 \%$ decrease in plant density.

2) Unequal selfed-seed inviability - Because inbreeding depression is expected to be weaker

306 in polyploids (Husband and Schemske 1997; Husband et al. 2008), we tested scenarios where the

307 probability of selfed-seed inviability for diploids was $k_{2}=0.9$, and for tetraploids $k_{4}$ took lower 
values in increments of 0.2 (minimum 0.1 ). For these $4 k_{2}-k_{4}$ pairs, we reran the equal and unequal life history scenarios described above with pollen-seed dispersal $d_{p}=d_{s}=0.5$ only (220 total parameter combinations).

3) Non-zero UG production - We consider values of unreduced gamete production (ug) by were rerun for a subset of the core dispersal-inviability parameters $\left(d_{p}=d_{s}=0.5\right.$, and $k_{2}=k_{4}=$ $0.1)$, for 275 total parameter combinations.

CLONAL REPRODUCTION SCENARIOS:

317 Here, we allow diploid and tetraploid ramet production to be non-zero; reproduction occurs both

318 sexually and clonally. In the equal clonality scenario, both diploids and tetraploids are perennial

$319\left(s v_{2}>0, s v_{4}>0\right)$, and produce an equal number of clonal ramets per generation $\left(c_{2}=c_{4}>0\right)$ at

320 some average distance away from the parent plant $\left(d_{C 2}=d_{C 4}>0\right)$. For both ramet production and

321 dispersal we consider two values: small vs. large genets $\left(c_{2}=c_{4}=1\right.$ or 5 ramets produced per

322 generation) and clumped vs. dispersed clonal architecture ( $d_{c 2}=d_{c 4}=0.5$ or 5 mean grid units).

323 All four combinations of clonal parameters were examined, hereafter referred to as clonal

324 strategies. For each strategy we include equal and unequal life history strategies, resulting in

3251620 parameter combinations over the core dispersal-inviability set.

We vary ramet production and clonal architecture separately to determine how differences in

327 clonality between cytotypes alters polyploid establishment, concentrating on equal life history

328 strategies only. Keeping ramet dispersal distance (i.e., clonal architecture) equal between

329 cytotypes $\left(d_{c 2}=d_{c 4}=0.5\right.$ or 5$)$, there are six combinations of $c_{2}$ and $c_{4}$, including $c_{2}=0$ or $c_{4}=0$,

330 for a total of 108 scenarios for the core dispersal-inviability parameter set. Similarly, keeping 
331 ramet production equal between cytotypes $\left(c_{2}=c_{4}=1\right.$ or 5$)$ while varying ramet dispersal results

332 in 18 additional combinations.

333 To test alternate model formulations for the clonal scenarios, we also consider:

334 1) Exponential ramet dispersal - Our model implicitly includes a competitive advantage to

335 clonal ramets over seedling recruits by assuming a normal Gaussian distribution for ramet

336 dispersal probability (eq. [2]; fig. S1B). We compared our main results with a model where

337 ramet dispersal follows an exponential density function (eq. [1]; fig. S1A), so that the

338 recruitment probability at a site $x$ grid units away from the parent plant is the same for both

339 sexual and clonal offspring. We reran the full set of equal clonality scenarios (1620 parameter

340 combinations).

341 2) Unequal selfed-seed inviability - We set the probability of selfed-seed inviability for

342 diploids at $k_{2}=0.9$, while for tetraploids $k_{4}$ took lower values in increments of 0.2 (minimum

3430.1 ). For these $4 k_{2}-k_{4}$ pairs, we reran the equal clonality and equal life history scenarios

344 described above, for ramet dispersal with either a Gaussian (432 parameter combinations) or

345 exponential probability function (432 parameter combinations).

347 MODEL OUTPUT:

348 Simulations were replicated 20 times for each of the 5175 unique sets of parameters we

349 investigated, for a total of 103500 model runs. A replicate could result in three possible

350 outcomes: exclusion of diploids (fixation of tetraploids), exclusion of tetraploids (fixation of

351 diploids), or a stable equilibrium that includes both cytotypes. Exclusion of diploids occurs once

352 tetraploids have replaced all diploids - because tetraploids cannot produce double-reduced

353 gametes, there is no way for diploids to re-enter the population. Complete exclusion of 
354 tetraploids can only occur when UG production is zero; otherwise a low equilibrium frequency

355 of tetraploids is expected as new tetraploid individuals are occasionally formed via UGs. In this

356 case, the stability of cytotype coexistence was assessed by examining average tetraploid

357 frequency over the last 100 generations of the simulation, and deviations of less than 10

358 individuals per generation over this period were deemed stable. One of the three final states was

359 generally reached within 100 generations, but we continued simulations for a maximum of 500

360 generations when unreduced gamete (UG) production was non-zero, to capture rare or delayed

361 events. To determine the probability of polyploid fixation for a particular parameter set, we

362 calculated the proportion of replicates that end in each state, and average the number of

363 generations needed to reach them.

364 For each simulation run, we tracked several demographic and reproductive metrics per

365 generation, including diploid and tetraploid survivorship, number and average size of diploid and

366 tetraploid individuals and genets, average local neighbourhood composition (defined as an

367 extended Moore neighbourhood 2 grid units in each dimension; total 24 surrounding cells not

368 including the focal cell), local neighbourhood vacancy (total 25 cells including the focal cell), the

369 total number of offspring of each sexual cross-type produced (table S1), the number of clonal

370 ramets produced, as well as the number of each offspring type successfully recruited into the

371 population.

372

\section{RESULTS}

\section{LIFE HISTORY:}

375 In models with equal survivorship $\left(s v_{2}=s v_{4}\right)$ between cytotypes and no clonal reproduction,

376 there was no successful establishment of tetraploids, unless other conditions were also met (e.g., 
$377 u g \neq 0)$. Under unequal survivorship $\left(s v_{2} \neq s v_{4}\right)$, tetraploid establishment only occurred under

378 short pollen and seed dispersal distances $\left(d_{p}=d_{s}=0.5\right.$, high autogamy). Establishment

379 probability increased when both diploids and tetraploids had high survival, and when tetraploids

380 had significant survival advantages over diploids (fig. 2A). When selfed-seed inviability was

381 high $\left(k_{2}=k_{4}=0.9\right)$, tetraploids needed a large survivorship advantage and a survival probability

382 of at least 0.8 to establish, regardless of diploid survival (fig. 2A). When selfed-seed inviability

383 was low $\left(k_{2}=k_{4}=0.1\right)$, tetraploids needed a minimum survival advantage of 0.4 when among

384 annual diploids $\left(s v_{2}=0\right)$, but only 0.2 when diploids had higher survival probabilities (fig. 2A).

385 Time to tetraploid fixation was more rapid with larger tetraploid survival advantages, but was not

386 affected by diploid survival probability; whereas tetraploid exclusion was significantly slower

387 when tetraploid survival advantage was large and when diploid survival was high (fig. S2).

388 Lower population densities expanded the parameter space that allowed successful tetraploid

389 establishment (fig. S3A), but tetraploid fixation or exclusion times were longer (figs. S3B, C).

390 Reducing the inviability of selfed-seed in tetraploids relative to diploids significantly increased

391 establishment probability (fig. 2B), even when cytotype survival was equal. Times to exclusion

392 were similar to the equal self-seed inviability simulations, but fixation times were significantly

393 faster (figs. S4A, B).

394 The rate of unreduced gamete (UG) production was positively associated with tetraploid

395 fixation probability, with the critical value being between $u g=0.15$ and $u g=0.2$ when cytotype

396 life history strategies were equal and pollen-seed dispersal distance was moderate to far $\left(d_{p}=d_{s}\right.$

$397=2$ or 5), and this threshold was reduced to $u g=0.1$ when $d_{p}=d_{s}=0.5$ and selfed-seed

398 inviability was low $\left(k_{2}=k_{4}=0.1\right.$; data not shown). When cytotypes varied in life history

399 strategy, tetraploids were always successful when $u g \geq 0.1$ (fig. S5A), unless diploid and 
400 tetraploid survival were both very high $\left(s v_{2}=s v_{4}=0.9\right)$, where 500 generations was just shy of

401 enough time for complete fixation (fig. S6A). When $u g=0.05$, a tetraploid survival advantage of

4020.2 resulted in tetraploid establishment across all diploid survival probabilities (fig. 2C), and

403 when tetraploid advantage was 0.1 establishment success was positively correlated with diploid

404 survival; low diploid survival resulted in tetraploid establishment only rarely, intermediate

405 survivals $\left(s v_{2}=0.3-0.4\right)$ showed a trend towards eventual tetraploid establishment (fig. S6A),

406 and $s v_{2} \geq 0.5$ always ended in tetraploid fixation in less than 500 generations (fig. 2C). Further

407 reducing $\mathrm{UG}$ production to $u g=0.01$ revealed a similar pattern (fig. $2 \mathrm{C}$, Figure 6A). For

408 simulations that did not proceed towards tetraploid fixation, both cytotypes coexisted with low

409 tetraploid frequencies of $\sim 10$ tetraploids per generation ( $1 \%$ of the population) for $u g=0.05$, and

$410 \sim 2(0.2 \%)$ when $u g=0.01$ (figs. S6A, B). In general, mean time to tetraploid fixation was slower

411 for more perennial populations, and when tetraploid survival advantage was small, but these

412 relationships were dependent on $u g$ value (fig. S5B).

414 CLONAL REPRODUCTION:

\section{Clonality and Life History Strategy:}

416 Tetraploid establishment probability was higher when individuals were able to reproduce both

417 sexually and clonally (fig. 3), but the effects of clonality were strongly dependent on genet size

418 and architecture (small vs. large genets, clumped vs. dispersed ramets). As in the nonclonal

419 scenarios, establishment increased with both diploid survival and tetraploid survival advantage,

420 but still no establishment occurred when cytotypes had equal life histories (figs. 3, S7). Large

421 dispersed clonal strategies had the highest establishment probabilities across parameter space

422 (fig. 3). Differences between clonal strategies were weaker when pollen and seed dispersal 
423 distances were short $\left(d_{p}=d_{s}=0.5\right.$, high autogamy, low geitonogamy), but when pollen and seed

424 dispersal was far $\left(d_{p}=d_{s}=5\right.$, low autogamy and high geitonogamy $)$ small and clumped genets

425 were substantially less successful than large and dispersed genets (fig. 3). Selfed-seed inviability

426 did not influence clonal tetraploid establishment probability (fig. S7), nor did lowering selfed-

427 seed inviability for tetraploids relative to diploids (fig. S8). Mean time to tetraploid fixation was

428 faster for all clonal strategies compared to the nonclonal, but there were no consistent differences

429 in exclusion time (fig. S9). The effects of life history strategy on establishment under the

430 alternate exponential formulation for ramet dispersal were nearly identical to the nonclonal

431 scenarios, but with faster time to fixation (fig. S10). Unlike under Gaussian ramet dispersal,

432 reducing tetraploid selfed-seed inviability compared to diploids increased establishment

433 probability (fig. S8).

\section{Divergent Clonal Strategies:}

436 Tetraploids with fewer ramets than diploids had no establishment success (fig. 4). When

437 tetraploid ramet production was higher, tetraploid establishment depended on clonal architecture, 438 pollen and seed dispersal distance, and life history strategy (fig. 4). Increasing pollen and seed

439 dispersal distances decreased establishment probability, particularly for clumped genets (fig. 4).

440 Tetraploids with greater survivorship tended to have lower establishment success when the

441 difference in genet size between cytotypes was large $\left(c_{2}=0, c_{4}=5\right.$; fig. 4$)$, but establishment

442 was positively correlated with survival when differences were small $\left(c_{2}=0, c_{4}=1\right)$ and genets

443 were dispersed (fig. 4). Selfed-seed inviability did not affect tetraploid establishment (results not 444 shown). 
Tetraploids with clumped clonal architecture could not establish among dispersed diploids,

446 but dispersed tetraploid genets generally had high establishment success among clumped

447 diploids (fig. S11). In the latter scenario, tetraploids with large genets were little affected by

448 pollen and seed dispersal distance, and establishment decreased with survival (fig. S11).

449 Conversely, for small genets establishment success decreased with increasing pollen and seed

450 dispersal distance (fig. S11). Here, increasing survival lowered establishment probability when

451 pollen and seed dispersal was restricted $\left(d_{p}=d_{s}=0.5\right)$, but enhanced it when pollen and seed

452 dispersal was far $\left(d_{p}=d_{s}=2\right.$ or 5, fig. S11). Selfed-seed inviability did not affect tetraploid

453 establishment when clonal architecture differed between cytotypes (results not shown).

The Mating and Demographic Consequences of Clonal Reproduction:

456 To illustrate the effects of different clonal strategies on patterns of tetraploid establishment, we

457 focus on the set of models where $s v_{2}=0.5, s v_{4}=0.9, d_{p}=d_{s}=0.5$ or $5, k_{2}=k_{4}=0.1$, and $u g=0$,

458 as this combination led to successful establishment in the majority of simulations. Diploids and

459 tetraploids have equal clonal strategies $\left(c_{2}=c_{4}, d_{C 2}=d_{C 4}\right)$.

$460 \quad$ Reproductive and clonal strategies substantially influenced the spatial structure of tetraploid

461 populations, even when overall establishment probabilities were similar (fig. 5A). In the

462 nonclonal scenarios, tetraploids spread into the diploid population in a tight circle, and at 30

463 generations only occupied $3-5 \%$ of the population (fig. 5B). In comparison, clonal tetraploids

464 established quicker, with clumped architectures expanded from localized points, and dispersed

465 architectures quickly establishing across the entire population lattice (fig. 5B). Low ramet

466 production promoted the establishment of many distinct tetraploid genets, but populations with

467 high ramet production were often dominated by a few very large genets (fig. 5B). 
Structural differences between clonal strategies affected patterns of within- vs. betweencytotype mating during the establishment process, and the timing and location of recruitment of

470 sexual vs. clonal offspring. For nonclonal tetraploids, when simulations began most ovules were

471 involved in self-fertilized autogamous or inviable intercytotype matings, and nearly all viable

472 offspring and recruits were autogamously produced (fig. 6A). As the simulations progressed and

473 tetraploids increased in number, intracytotype matings and recruits became more common (fig.

$4746 \mathrm{~A})$

475 All clonal tetraploids experienced early increases in geitonogamous mating (fig. 6B) and

476 geitonogamous seed production (fig. 6C). When pollen and seed dispersal were restricted $\left(d_{p}=d_{s}\right.$

$477=0.5)$ autogamy was prevalent, though geitonogamy remained high for large and dispersed

478 strategies until tetraploid fixation, whereas outcrossed mating was more frequent in small and

479 clumped genets (fig. 6B). In contrast, when pollen and seed dispersal distances were far $\left(d_{p}=d_{s}\right.$

$480=5$ ) geitonogamous mating was dominant early during establishment, but intercytotype mating

481 overtook geitonogamy before fixation for all but the large dispersed clonal strategy (fig. 6B).

482 Sexual offspring were rarely recruited in the clonal scenarios, even when they were more

483 abundant than ramets (figs. 6C, D). Seedling recruitment frequencies mirrored those of the viable

484 seedlings available (fig. 6C), and was more common late during the establishment process, under

485 far pollen and seed dispersal distances $\left(d_{p}=d_{s}=5\right)$, and for small and clumped clonal

486 architectures (fig. 6D).

487 Under the alternate exponential formulation for ramet dispersal, tetraploid establishment was

488 nearly identical to the nonclonal scenario; sexual offspring recruited were mainly the result of

489 autogamy, and geitonogamy played little to no role (fig. S12). However, clonal ramet 
recruitment spiked early in establishment, particularly for large and dispersed strategies, but then

491 remained at a low frequency until tetraploid fixation (fig. S12).

492

\section{DISCUSSION}

494 Our results indicate that a perennial life history strategy and clonal reproduction can increase

495 polyploid establishment potential. However, we find that this is only the case when polyploids

496 have some advantage over diploids (e.g., higher per generation survival), or when unreduced

497 gamete production is high, suggesting that the ability to perennate or to produce clonal ramets

498 may not be enough to overcome Minority Cytotype Exclusion (MCE) in some circumstances. By

499 investigating how various clonal and life history strategies affects the spatial structure,

500 demography, and mating patterns within a mixed-ploidy population, we identify mechanisms

501 that can influence the spread of polyploids into a diploid population. Our model is the first to

502 incorporate the spatial attributes of clonal architecture and within-genet geitonogamous self-

503 fertilization, and we find that clonal strategy has a significant effect on the polyploid

504 establishment process, and major consequences for polyploid population structure.

505

506

507

508

509

510

511

512 2019), which highlight the importance of spatial structure, temporal dynamics, and chance

513 during the first few generations after a neopolyploid arises.

\section{PERENNIALITY PROMOTES POLYPLOIDY:}

Perennial polyploids should experience weaker Minority Cytotype Exclusion (MCE) pressure than annual polyploids because lengthening lifespan increases the chances of reproductive success via self-fertilization, and reduces stochastic loss of all polyploids from the population.

Our model supports these hypotheses and provides mechanistic links behind the broad-scale associations between polyploids and perenniality (Rice et al. 2019; Van Drunen and Husband 
We find that polyploids are more likely to succeed among longer-lived diploid species,

515 especially if perennial diploids give rise to polyploids with slightly longer lifespans. Notably, the

516 polyploid survival advantage needed for consistently successful establishment decreased with

517 increasing diploid perenniality (fig. 2A). This pattern was driven by the demographic

518 consequences of 1) competition for space in the population, and 2) drawn-out polyploid

519 persistence when polyploids, or both cytotypes, were long-lived. When diploids are short-lived,

520 recruitment sites open up close to the rapidly-cycling diploids, which precludes the initial

521 recruitment of polyploid offspring and increases the likelihood of stochastic loss of all polyploids

522 unless they have very high relative survivorship. In contrast, when diploids and polyploids are

523 both long-lived there is significantly slower population turnover, longer polyploid persistence,

524 and more potential for polyploid offspring recruitment. However, when population density is low

525 the conditions under which polyploids can establish are greater (fig. S3). This suggests that in

526 less competitive environments, polyploid success may be less dependent on the life history

527 strategy of diploids, in line with previous models showing that ecological niche (Fowler and

528 Levin 1984; Rodríguez 1996) and physical (Griswold 2021) segregation can facilitate polyploid

529 establishment, even when individuals have an annual life history strategy.

530 Interactions between the spatial and demographic effects of divergent life history strategies

531 in mixed-cytotype populations offers a reason why polyploidy is more common in perennial

532 species - the $\geq 40 \%$ increase in survivorship needed to establish among short-lived diploids in

533 our model (fig. 2A) may be an unfeasibly large shift in neopolyploids, whereas a survival

534 probability increase of $20 \%$ among long-lived diploids may be more tenable. There are numerous

535 examples of naturally occurring polyploids that exhibit a life history shift compared to their

536 diploid relatives. For example, in the mixed-ploidy species Centaurea stoebe tetraploids are 
537 iteroparous short-lived perennials while diploids are predominantly semelparous annuals (Mráz

538 et al. 2012), and tetraploid cultivars of Nasturtium officinale perennate more easily than diploid

539 lines (Manton 1935). But few studies have addressed life history changes in perenniality in

540 neopolyploids (but see Müntzing 1936), limiting our understanding of the strength of the

541 immediate effects of WGD and phenotypic differences between diploids and polyploids, and the

542 consequences for polyploid establishment in natural populations. Indeed, here we demonstrate

543 that autopolyploids are unable to establish among diploids with equal life history strategies

544 unless they have additional advantages (e.g., significantly lower inbreeding depression), a result

545 corroborated by previous models (Rodríguez 1996; Chrtek et al. 2017; Spoelhof et al. 2020).

546 Polyploid persistence time was prolonged when diploids and polyploids were highly

547 perennial, considerably slowing model dynamics by decreasing polyploid death and recruitment

548 rates (see also Chrtek et al. 2017; Spoelhof et al. 2020). Surveys in natural populations have

549 found that cytotype frequencies in annual species can fluctuate wildly between growing seasons,

550 sometimes leading to the complete loss of diploids or polyploids within a population (Čertner et

551 al. 2017), whereas cytotype coexistence in perennial species may be maintained over many

552 generations (Lumaret et al. 1987; Keeler 2004; Kao and Parker 2010). Thus, perenniality has

553 been invoked as a non-adaptive factor promoting cytotype coexistence in nature (Duchoslav et

554 al. 2010; Hanzl et al. 2014; Hanušová et al. 2019), though it is often unknown whether mixed-

555 ploidy populations are in stable states, or represent only short snapshots of ongoing competition

556 and exclusion (Kolář et al. 2017). Our results suggest that perenniality may contribute to an

557 illusion of stable coexistence for a much longer timeframe than field studies typically encompass

558 (e.g., (e.g., Čertner et al. 2017; but see Buggs and Pannell 2006). For some perennial scenarios

559 both cytotypes coexisted for more than 300 generations while moving steadily towards polyploid 
560 fixation or exclusion (fig. S2), whereas annual polyploids were typically excluded in 1 or 2

561 generations. Either end of this temporal spectrum presents challenges for studying neopolyploid

562 establishment in natural populations, emphasizing the utility of theory in understanding this

563 process.

564 An extended period of close interaction between perennial diploids and polyploids may have 565 significant consequences for polyploid evolution during and after establishment. For example, ongoing intercytotype reproductive events could result in selection for assortative mating,

567 driving divergence in floral morphology or phenology between diploids and polyploids (Oswald 568 and Nuismer 2011; Husband et al. 2016). Prolonged intercytotype mating will also promote 569 polyploid establishment when diploid unreduced gamete (UG) production is nonzero, because 570 the probability of two UGs giving rise to a viable polyploid offspring in a diploid-diploid cross is $571 u g^{2}$, but the probability of one UG being involved in a diploid-polyploid cross is $u g$ (table S1).

572 Our model is the first to incorporate UG production and perenniality, and we find that a UG 573 production rate of $1-5 \%$ is sufficient to ensure polyploid success when diploids and polyploids 574 are perennial, providing polyploids also have a small survival advantage (fig. 2C). This is similar 575 to estimates of UG frequencies in natural populations (0.05-2\%; Ramsey and Schemske 1998;

576 Kreiner et al. 2017), and is substantially lower than the UG production rate of $\sim 17 \%$ required for 577 non-selfing annuals with random mating (Felber 1991; Husband 2004), or 10\% for annuals with 578 self-fertilization and low inbreeding depression (Rausch and Morgan 2005). UG production may 579 then be less of a limiting step for polyploid formation among perennials where reproductive 580 events are spread over multiple generations. 
583 When all else is equal between cytotypes, simply being clonal is not enough to ensure

584 establishment success under any scenario we investigated - some small advantage over diploids

585 is needed (e.g., higher ramet production). In our model, increased clonality confers high

586 establishment success, as in mixed diploid-tetraploid Chamerion angustifolium (fireweed), where

587 neotetraploids exhibit immediate increases in clonal rootbud production (Van Drunen and

588 Husband 2018b). Conversely, decreased clonal investment in polyploids results in no

589 establishment (fig. 4), a pattern consistent with the lack of natural cytotype variation in Fragaria

590 vesca (woodland strawberry), where artificial neotetraploids have lower stolon production (Van

591 Drunen and Husband 2018a). These remain the only species in which neopolyploid investment

592 in clonal reproduction has been measured, but if this pattern applies more generally, it would

593 suggest that the production of clonal ramets is an important factor in polyploid establishment

594 (Spoelhof et al. 2017).

595 Perenniality and clonal reproduction have both been implicated in polyploid success in 596 natural populations (Rice et al. 2019; Van Drunen and Husband 2019), but few studies have 597 contrasted their importance in the early stages of polyploid evolution. Here, we show that 598 polyploids with a combination of perenniality and clonal reproduction generally have greater 599 establishment success than polyploids that are strictly sexual. In some scenarios, perenniality 600 alone was enough to ensure polyploid establishment (e.g., when autogamy was high). While 601 clonal reproduction did not affect final establishment probability in these simulations, it still 602 significantly altered the establishment process. For instance, polyploids were able to spread into 603 the diploid population faster when clonal, even when seedlings and ramets were equal 604 competitors (fig. S10), but this effect was heavily moderated by clonal strategy. Spoelhof et al. 605 (2020) speculated that rapid clonal expansion may increase establishment probability, but result 
in genetically homogeneous polyploid populations. We confirm this outcome, particularly for

607 clonal strategies that produce many ramets with high lateral spread (fig. 5B). Here, it is possible

608 that polyploids may enjoy short-term success, but may not survive in the long-term if the lack of

609 genetic variability in the polyploid population leads to reduced evolutionary potential (Muller

610 1932; Pan and Price 2002; Barrett 2015; Herben et al. 2016). According to our results, polyploids

611 conforming to a large-clumped clonal architecture may be the most successful if they spread

612 quickly while maintaining higher genet diversity than polyploids with more dispersible ramets

613 (fig. 5B). Nonetheless, spreading architectures may perform well in environments with high

614 temporal variability or frequent disturbance where slower expansion could result in the stochastic

615 loss of all polyploids (Čertner et al. 2017), especially if genetic uniformity is counteracted by

616 recurrent polyploid formation, immigration, or high rates of vegetative somatic mutation

617 between ramets (Yu et al. 2020).

618 Our model demonstrates that spatial clonal architecture has a large effect on the polyploid

619 establishment process, but that no single clonal strategy is superior under all circumstances.

620 Similarly, phylogenetic comparative studies have found mixed support for associations between

621 particular clonal strategies and polyploid occurrence, depending on the group of species being

622 studied (Herben et al. 2017; Van Drunen and Husband 2019). Overall, given the wide diversity,

623 evolutionary lability, and multi-functional nature of clonal modes across the angiosperms

624 (Herben and Klimešová 2020), it seems unlikely that there is a one-size-fits-all hypothesis

625 describing the relationship between clonal reproduction and polyploidy. For example, bulbs or

626 corms (generally “clumped” clonal architectures) are involved in resource storage (Klimeš et al.

627 1997), which may affect over-winter survival or the timing and extent of sexual reproduction in

628 the next growing season. Thus, polyploid establishment may indeed be successful due to 
629 clonality in species with these strategies, but for significantly different reasons than explored in

630 our current model.

\section{CLONAL ARCHITECTURE \& MATING:}

633 Clonal reproduction may enhance polyploid establishment by creating local polyploid majorities

634 (Baack 2005; Spoelhof et al. 2020), but previous models have overlooked one of the key aspects

635 of this polyploidy-promoting mechanism: geitonogamous self-fertilization. Geitonogamous self-

636 fertilization between different flowers on the same plant, or between clonal ramets, is often

637 regarded as a negative and non-adaptive side-effect of outcrossing (Lloyd 1992; Eckert 2000).

638 Geitonogamy generally involves mating costs, as it requires the same pollination process as

639 would occur with outcrossing, but it reduces outcross siring success and results in pollen (Harder

640 and Barrett 1995; Lau et al. 2008) and seed discounting (Lloyd 1992) when there is any

641 inbreeding depression. But geitonogamy is rarely considered in the complete absence of

642 compatible mates, where self-fertilization may be the only recourse for producing viable sexual

643 offspring. Our model shows that geitonogamous self-fertilization between clonal ramets can

644 confer a significant fitness advantage to establishing polyploids by enabling them to acquire

645 same-cytotype mates, revealing a situation where geitonogamy might be adaptive. Here, when a

646 new polyploid is instantly reproductively isolated from its progenitors, the costs typically

647 associated with pollen and seed discounting via geitonogamy instead apply to intercytotype

648 outcrossing that does not produce viable recruits.

649 Rates of geitonogamy are expected to vary with clonal architecture (Charpentier 2002;

650 Matsuo et al. 2014; Van Drunen et al. 2015). We can clearly see this in our model, where the

651 long reach of dispersed polyploid genets enables ramet recruitment over a wide area, leading to 
652 large genets and high rates of geitonogamy. In contrast, seedlings are equal or better dispersers

653 than ramets when clonal architecture is clumped (fig. S1), resulting in smaller genets, more

654 sexual recruits, and more frequent intracytotype outcrossing than geitonogamous self-

655 fertilization (fig. 6). These mating differences contribute to establishment speed and overall

656 establishment probability, especially when pollen and seed dispersal distances are long and

657 sexual reproduction is not dominated by autogamy. Across all clonal strategies, however,

658 geitonogamy substantially reduced ovule waste to intercytotype mating early in the

659 establishment process (fig. 6).

660 We might then predict that neopolyploids will experience strong selection on traits that

661 increase geitonogamy, such as ramet production (Hu et al. 2015), clonal architecture (Van

662 Drunen and Husband 2018a), or floral display size (Vamosi et al. 2007). But as polyploids

663 spread throughout the population and intracytotype outcrossing becomes more probably,

664 selection may reverse and instead favour outcross sexual reproduction. There is some evidence

665 for this occurring in autotetraploid Chamerion angustifolium, where initial WGD-driven

666 increases in synthetic neotetraploid rootbud production is significantly reduced in naturally-

667 occurring tetraploids (Van Drunen and Husband 2018b; Walczyk and Hersch-Green 2019). In

668 our model we saw a shift towards recruiting outcrossed seedlings as polyploids became common

669 (fig. 6), but we could not evaluate selection on reproductive strategy directly because we did not

670 include phenotypic trait evolution. Future work that allows for heritable variation in reproductive

671 strategy and sex allocation, and resource-based trade-offs between reproductive modes could

672 therefore be particularly insightful.

673 Clonal reproduction and geitonogamous self-fertilization may be less likely to facilitate

674 polyploid success when pollen is limited and pollen discounting is high (Yamauchi 2006), or 
675 when ramet recruitment into the population is low. In our model, we assumed that clonal ramets

676 were better competitors than seedlings, and so polyploid ramets were recruited much more often

677 than sexual offspring, even when they were less common in the recruitment pool (fig. 6). In

678 contrast, when ramets and seedlings were equal competitors in the exponential ramet dispersal

679 scenarios there were no differences in establishment probability between clonal and nonclonal

680 polyploids, though fixation times were still substantially shorter (figs. S8, S10). Ramet

681 competitive superiority is realistic for most clonal species, where ramet recruits tend to be more

682 common than seedlings within populations (Eriksson 1993; Vallejo-Marín et al. 2010;

683 Vandepitte et al. 2010; Johnson et al. 2020), but the validity of this assumption may vary for

684 different taxa, and ecological or demographic conditions. For instance, considering only within-

685 population processes ignores the role of seedlings in long-distance colonisation events and meta-

686 populations dynamics (Winkler and Fischer 2001; Griswold 2021; but see Scherrer et al. 2017),

687 and thus polyploids arising from diploids that occur in patchy habitats might benefit from more

688 dispersible sexual offspring than locally-restricted clonal propagules.

690 CONCLUSIONS:

691 Whether perenniality and clonal reproduction promote polyploid establishment depends on 692 multiple factors, including the phenotypic effects of WGD, local habitat availability, clonal 693 strategy, mating patterns and pollination system, inbreeding depression, and more. Ultimately, 694 determining whether the processes in our model occur in natural populations will require fine695 scale genetic data and extensive sampling, and may not be possible in polyploid systems that are 696 long established. Further models exploring a variety of situations (e.g., clonal vs. sexual 697 reproduction and polyploid establishment in patchy habitats), and experimental studies involving 
synthetic neopolyploids or very young natural neopolyploids (e.g., tetraploid Mimulus guttatus in the Shetland Isles; Simón-Porcar et al. 2017) will be key to unraveling the individual and interactive roles of perenniality and clonality in early polyploid evolution.

\section{ACKNOWLEDGEMENTS}

703 We thank B.C. Husband for input on study concept. Funding for this study was provided by the

704 Natural Sciences and Engineering Research Council (NSERC) through a Postdoctoral

705 Fellowship to W.E.V., and a Discovery Grant to J.F.

DATA AND CODE AVAILABILITY

All of the code needed to run the simulation model in this article is available online at:

https://github.com/wevandrunen/autopolyploid-establishment-lifehistory-clonalarchitecture.

\section{AUTHOR CONTRIBUTION STATEMENT}

712 W.E.V. and J.F. conceived of the study. W.E.V. developed the model, and analyzed the output.

713 W.E.V. wrote the manuscript with input from J.F.

\section{LITERATURE CITED}

716 Anneberg, T. J., and K. A. Segraves. 2020. Nutrient enrichment and neopolyploidy interact to

717 increase lifetime fitness of Arabidopsis thaliana. Plant and Soil 456:439-453.

718 Baack, E. J. 2005. To succeed globally, disperse locally: effects of local pollen and seed 719 dispersal on tetraploid establishment. Heredity 94:538-546.

720 Baack, E., M. C. Melo, L. H. Rieseberg, and D. Ortiz-Barrientos. 2015. The origins of reproductive isolation in plants. New Phytologist 207:968-984. 
Baldwin, S. J., and B. C. Husband. 2013. The association between polyploidy and clonal reproduction in diploid and tetraploid Chamerion angustifolium. Molecular Ecology 22:18061819.

Barabás, G., R. D’Andrea, and S. M. Stump. 2018. Chesson's coexistence theory. Ecological Monographs 88:277-303.

Barrett, S. C. H. 2015. Influences of clonality on plant sexual reproduction. Proceedings of the National Academy of Sciences 112:8859-8866.

Beaulieu, J. M., I. J. Leitch, S. Patel, A. Pendharkar, and C. A. Knight. 2008. Genome size is a strong predictor of cell size and stomatal density in angiosperms. New Phytologist 179:975986.

Bennett, M. D. 1972. Nuclear DNA content and minimum generation time in herbaceous plants. Proceedings of the Royal Society of London. Series B, Biological Sciences 181:109-135.

Blomme, J., D. Inzé, and N. Gonzalez. 2014. The cell-cycle interactome: a source of growth regulators? Journal of Experimental Botany 65:2715-2730.

Buggs, R. J. A., and J. R. Pannell. 2006. Rapid displacement of a monoecious plant lineage is due to pollen swamping by a dioecious relative. Current Biology 16:996-1000.

Burton, T. L., and B. C. Husband. 2001. Fecundity and offspring ploidy in matings among diploid, triploid and tetraploid Chamerion angustifolium (Onagraceae): consequences for tetraploid establishment. Heredity 87:573-582.

Čertner, M., E. Fenclová, P. Kúr, F. Kolář, P. Koutecký, A. Krahulcová, and J. Suda. 2017. Evolutionary dynamics of mixed-ploidy populations in an annual herb: dispersal, local persistence and recurrent origins of polyploids. Annals of Botany 120:303-315.

Charpentier, A. 2002. Consequences of clonal growth for plant mating. Evolutionary Ecology 15:521-530.

Chrtek, J., T. Herben, R. Rosenbaumová, Z. Münzbergová, Z. Dočkalová, J. Zahradníček, J. Krejčíková, et al. 2017. Cytotype coexistence in the field cannot be explained by intercytotype hybridization alone: linking experiments and computer simulations in the sexual species Pilosella echioides (Asteraceae). BMC Evolutionary Biology 17:87.

Duchoslav, M., L. Šafářová, and F. Krahulec. 2010. Complex distribution patterns, ecology and coexistence of ploidy levels of Allium oleraceum (Alliaceae) in the Czech Republic. Annals of Botany 105:719-735.

Eckert, C. G. 2000. Contributions of autogamy and geitonogamy to self-fertilization in a massflowering, clonal plant. Ecology 81:532-542.

Eriksson, O. 1993. Dynamics of genets in clonal plants. Trends in Ecology \& Evolution 8:313316. 
Felber, F. 1991. Establishment of a tetraploid cytotype in a diploid population: Effect of relative fitness of the cytotypes. Journal of Evolutionary Biology 4:195-207.

Felber, F., and J. D. Bever. 1997. Effect of triploid fitness on the coexistence of diploids and tetraploids. Biological Journal of the Linnean Society 60:95-106.

Fowler, N. L., and D. A. Levin. 1984. Ecological constraints on the establishment of a novel polyploid in competition with Its diploid progenitor. The American Naturalist 124:703-711.

Fowler, N. L., and D. A. Levin. 2016. Critical factors in the establishment of allopolyploids. American Journal of Botany 103:1236-1251.

Griswold, C. K. 2021. The effects of migration load, selfing, inbreeding depression, and the genetics of adaptation on autotetraploid versus diploid establishment in peripheral habitats. Evolution 75:39-55.

Gustafsson, Å. 1948. Polyploidy, life-form and vegetative reproduction. Hereditas 34:1-22.

Handel, S. N. 1985. The intrusion of clonal growth patterns on plant breeding systems. The American Naturalist 125:367-384.

Hanušová, K., M. Čertner, T. Urfus, P. Koutecký, J. Košnar, C. J. Rothfels, V. Jarolímová, et al. 2019. Widespread co-occurrence of multiple ploidy levels in fragile ferns (Cystopteris fragilis complex; Cystopteridaceae) probably stems from similar ecology of cytotypes, their efficient dispersal and inter-ploidy hybridization. Annals of Botany 123:845-855.

Hanzl, M., F. Kolár̆, D. Nováková, and J. Suda. 2014. Nonadaptive processes governing early stages of polyploid evolution: Insights from a primary contact zone of relict serpentine Knautia arvensis (Caprifoliaceae). American Journal of Botany 101:935-945.

Harder, L. D., and S. C. H. Barrett. 1995. Mating cost of large floral displays in hermaphrodite plants. Nature 373:512-515.

Herben, T., and J. Klimešová. 2020. Evolution of clonal growth forms in angiosperms. New Phytologist 225:999-1010.

Herben, T., J. Suda, and J. Klimešová. 2017. Polyploid species rely on vegetative reproduction more than diploids: a re-examination of the old hypothesis. Annals of Botany 120:341-349. plants: correlated syndromes or independent strategies? (M. Rees, ed.)Journal of Ecology 104:1696-1706.

Hroudová, Z., and P. Zákravský. 1993. Ecology of two cytotypes of Butomus umbellatus II. Reproduction, growth and biomass production. Folia Geobotanica \& Phytotaxonomica

789 28:413-424. 
Hu, Y., S. C. H. Barrett, D. Zhang, and W. Liao. 2015. Experimental analysis of mating patterns in a clonal plant reveals contrasting modes of self-pollination. Ecology and Evolution 5:54235431.

Husband, B. C. 2000. Constraints on polyploid evolution: A test of the minority cytotype exclusion principle. Proceedings: Biological Sciences 267:217-223.

. 2004. The role of triploid hybrids in the evolutionary dynamics of mixed-ploidy populations. Biological Journal of the Linnean Society 82:537-546.

Husband, B. C., S. J. Baldwin, and H. A. Sabara. 2016. Direct vs. indirect effects of wholegenome duplication on prezygotic isolation in Chamerion angustifolium : Implications for rapid speciation. American Journal of Botany 103:1259-1271.

Husband, B. C., S. J. Baldwin, and J. Suda. 2013. The incidence of polyploidy in natural plant populations: Major patterns and evolutionary processes. Pages 255-276 in J. Greilhuber, J. Dolezel, and J. F. Wendel, eds. Plant Genome Diversity Volume 2. Springer Vienna, Vienna.

Husband, B. C., B. Ozimec, S. L. Martin, and L. Pollock. 2008. Mating consequences of polyploid evolution in flowering plants: Current trends and insights from synthetic polyploids. International Journal of Plant Sciences 169:195-206.

Husband, B. C., and D. W. Schemske. 1997. The effect of inbreeding in diploid and tetraploid populations of Epilobium angustifolium (Onagraceae): Implications for the genetic basis of inbreeding depression. Evolution 51:737.

Johnson, A. J., R. J. Orth, and K. A. Moore. 2020. The role of sexual reproduction in the maintenance of established Zostera marina meadows. (C. Angelini, ed.)Journal of Ecology 108:945-957.

Kao, R. H., and I. M. Parker. 2010. Coexisting cytotypes of Arnica cordifolia: Morphological differentiation and local-scale distribution. International Journal of Plant Sciences 171:81-89.

Keeler, K. H. 2004. Impact of intraspecific polyploidy in $<i>$ Andropogon gerardii $<i />$ (Poaceae) populations. The American Midland Naturalist 152:63-74.

Klimeš, L., J. Klimešová, R. Hendricks, and J. van Groenendael. 1997. Clonal plant architecture: a comparative analysis of form and function. in H. de Kroon and J. van Groenendael, eds. The ecology and evolution of clonal plants. Backhuys, Leiden, the Netherlands.

Kolář, F., M. Čertner, J. Suda, P. Schönswetter, and B. C. Husband. 2017. Mixed-ploidy species: Progress and opportunities in polyploid research. Trends in Plant Science 22:1041-1055.

Kreiner, J. M., P. Kron, and B. C. Husband. 2017. Evolutionary dynamics of unreduced gametes. Trends in Genetics 33:583-593. 
Lau, J. A., R. E. Miller, and M. D. Rausher. 2008. Selection through male function favors smaller floral display size in the common morning glory Ipomoea purpurea (Convolvulaceae). The American Naturalist 172:63-74.

Levin, D. A. 1975. Minority cytotype exclusion in local plant populationsS. TAXON 24:35-43.

Levin, D. A. 2002. The role of chromosomal change in plant evolution. Oxford University Press.

Li, B.-H., X.-M. Xu, and M. S. Ridout. 2004. Modelling the establishment and spread of autotetraploid plants in a spatially heterogeneous environment: Establishment and spread of autotetraploid plants. Journal of Evolutionary Biology 17:562-573.

Lloyd, D. G. 1992. Self- and cross-fertilization in plants. II. The selection of self-fertilization. International Journal of Plant Sciences 153:370-380.

Lumaret, R., J.-L. Guillerm, J. Delay, A. Ait Lhaj Loutfi, J. Izco, and M. Jay. 1987. Polyploidy and habitat differentiation in Dactylis glomerata L. from Galicia (Spain). Oecologia 73:436446.

Maherali, H., A. E. Walden, and B. C. Husband. 2009. Genome duplication and the evolution of physiological responses to water stress. New Phytologist 184:721-731.

Manton, I. 1935. The cytological history of Watercress (Nasturtium officinale R. Br.). Zeitschrift für induktive Abstammungs- und Vererbungslehre 69:132-157.

Matsuo, A., H. Tomimatsu, J.-I. Suzuki, T. Saitoh, S. Shibata, A. Makita, and Y. Suyama. 2014. Female and male fitness consequences of clonal growth in a dwarf bamboo population with a high degree of clonal intermingling. Annals of Botany 114:1035-1041.

Mráz, P., S. Španiel, A. Keller, G. Bowmann, A. Farkas, B. Šingliarová, R. P. Rohr, et al. 2012. Anthropogenic disturbance as a driver of microspatial and microhabitat segregation of cytotypes of Centaurea stoebe and cytotype interactions in secondary contact zones. Annals of Botany 110:615-627.

Muller, H. J. 1932. Some genetic aspects of sex. The American Naturalist 66:118-138.

Müntzing, A. 1936. The evolutionary significance of autopolyploidy. Hereditas 21:363-378.

Nakayama, Y., H. Seno, and H. Matsuda. 2002. A population dynamic model for facultative agamosperms. Journal of Theoretical Biology 215:253-262.

Oswald, B. P., and S. L. Nuismer. 2011. A unified model of autopolyploid establishment and evolution. The American Naturalist 178:687-700.

Otto, S. P., and J. Whitton. 2000. Polyploid incidence and evolution. Annual Review of Genetics 34:401-437. 
Pan, J. J., and J. S. Price. 2002. Fitness and evolution in clonal plants: the impact of clonal growth. Pages 361-378 in J. F. Stuefer, B. Erschbamer, H. Huber, and J.-I. Suzuki, eds. Ecology and Evolutionary Biology of Clonal Plants. Springer Netherlands, Dordrecht.

Pannell, J. R., J. R. Auld, Y. Brandvain, M. Burd, J. W. Busch, P. Cheptou, J. K. Conner, et al. 2015. The scope of Baker's law. New Phytologist 208:656-667.

R Development Core Team. 2019. R: a language and environment for statistical computing (v.3.6.2). Vienna, Austria: R Foundation for Statistical Computing.

Ramsey, J., and T. S. Ramsey. 2014. Ecological studies of polyploidy in the 100 years following its discovery. Philosophical Transactions of the Royal Society B: Biological Sciences 369:20130352.

Ramsey, J., and D. W. Schemske. 1998. Pathways, mechanisms, and rates of polyploid formation in flowering plants. Annual Review of Ecology and Systematics 29:467-501.

- 2002. Neopolyploidy in flowering plants. Annual Review of Ecology and Systematics 3:589-639.

Rausch, J. H., and M. T. Morgan. 2005. The effect of self-fertilization, inbreeding depression, and population size on autopolyploid establishment. Evolution 59:1867-1875.

Rice, A., P. Šmarda, M. Novosolov, M. Drori, L. Glick, N. Sabath, S. Meiri, et al. 2019. The global biogeography of polyploid plants. Nature Ecology \& Evolution 3:265-273.

Rodríguez, D. J. 1996. A model for the establishment of polyploidy in plants: Viable but infertile hybrids, iteroparity, and demographic stochasticity. Journal of Theoretical Biology 180:189196.

Scherrer, D., P. Stoll, and J. Stöcklin. 2017. Colonization dynamics of a clonal pioneer plant on a glacier foreland inferred from spatially explicit and size-structured matrix models. Folia Geobotanica 52:353-366.

Simón-Porcar, V. I., J. L. Silva, S. Meeus, J. D. Higgins, and M. Vallejo-Marín. 2017. Recent autopolyploidization in a naturalized population of Mimulus guttatus (Phrymaceae). Botanical Journal of the Linnean Society.

Soltis, D. E., B. B. Misra, S. Shan, S. Chen, and P. S. Soltis. 2016a. Polyploidy and the proteome. Biochimica et Biophysica Acta (BBA) - Proteins and Proteomics 1864:896-907.

Soltis, D. E., C. J. Visger, D. B. Marchant, and P. S. Soltis. 2016b. Polyploidy: Pitfalls and paths to a paradigm. American Journal of Botany 103:1146-1166.

Spoelhof, J. P., D. E. Soltis, and P. S. Soltis. 2020. Habitat shape affects polyploid establishment in a spatial, stochastic model. Frontiers in Plant Science 11:592356. 
Spoelhof, J. P., P. S. Soltis, and D. E. Soltis. 2017. Pure polyploidy: Closing the gaps in autopolyploid research. Journal of Systematics and Evolution 55:340-352.

Stebbins, G. L. 1950. Variation and evolution in plants. Columbia University Press, New York.

Sutherland, B. L., T. Miranda-Katz, and L. F. Galloway. 2020. Strength in numbers? Cytotype frequency mediates effect of reproductive barriers in mixed-ploidy arrays. Evolution $74: 2281-2292$.

Thompson, J. D., and R. Lumaret. 1992. The evolutionary dynamics of polyploid plants: Origins, establishment and persistence. Trends in Ecology and Evolution 7:302-307.

Vallejo-Marín, M., M. E. Dorken, and S. C. H. Barrett. 2010. The ecological and evolutionary consequences of clonality for plant mating. Annual Review of Ecology, Evolution, and Systematics 41:193-213.

Vamosi, J. C., S. J. Goring, B. F. Kennedy, R. J. Mayberry, C. M. Moray, L. A. Neame, N. D. Tunbridge, et al. 2007. Pollination, floral display, and the ecological correlates of polyploidy. Functional Ecosystems and Communities 1:1-9.

Van Drunen, W. E., and B. C. Husband. 2018a. Whole-genome duplication decreases clonal stolon production and genet size in the wild strawberry Fragaria vesca. American Journal of Botany 105:1712-1724.

Van Drunen, W. E., and B. C. Husband. 2018b. Immediate vs. evolutionary consequences of polyploidy on clonal reproduction in an autopolyploid plant. Annals of Botany 122:195-205.

Van Drunen, W. E., and B. C. Husband. 2019. Evolutionary associations between polyploidy, clonal reproduction, and perenniality in the angiosperms. New Phytologist 224:1266-1277.

Van Drunen, W. E., M. van Kleunen, and M. E. Dorken. 2015. Consequences of clonality for sexual fitness: Clonal expansion enhances fitness under spatially restricted dispersal. Proceedings of the National Academy of Sciences 112:8929-8936.

Vandepitte, K., T. De Meyer, H. Jacquemyn, I. Roldán-Ruiz, and O. Honnay. 2013. The impact of extensive clonal growth on fine-scale mating patterns: a full paternity analysis of a lily-ofthe-valley population (Convallaria majalis). Annals of Botany 111:623-628.

Vandepitte, K., I. Roldán-Ruiz, H. Jacquemyn, and O. Honnay. 2010. Extremely low genotypic diversity and sexual reproduction in isolated populations of the self-incompatible lily-of-thevalley (Convallaria majalis) and the role of the local forest environment. Annals of Botany 105:769-776.

Walczyk, A. M., and E. I. Hersch-Green. 2019. Impacts of soil nitrogen and phosphorus levels on cytotype performance of the circumboreal herb Chamerion angustifolium: implications for polyploid establishment. American Journal of Botany 106:906-921. 
922 Winkler, E., and M. Fischer. 2001. The role of vegetative spread and seed dispersal for optimal 923 life histories of clonal plants: a simulation study. Evolutionary Ecology 15:281-301.

924 Winkler, E., and J. Stöcklin. 2002. Sexual and vegetative reproduction of Hieracium pilosella L. under competition and disturbance: A grid-based simulation model. Annals of Botany

927 Wood, T. E., N. Takebayashi, M. S. Barker, I. Mayrose, P. B. Greenspoon, and L. H. Rieseberg. 928 2009. The frequency of polyploid speciation in vascular plants. Proceedings of the National 929 Academy of Sciences 106:13875-13879.

930 Yamauchi, A. 2006. Formulation of competition between sexual and selfing forms. Evolution $931 \quad 60: 1952-1953$.

932 Yamauchi, A., A. Hosokawa, H. Nagata, and M. Shimoda. 2004. Triploid bridge and role of 933 parthenogenesis in the evolution of autopolyploidy. The American Naturalist 164:101-112.

934 Yu, L., C. Boström, S. Franzenburg, T. Bayer, T. Dagan, and T. B. H. Reusch. 2020. Somatic genetic drift and multilevel selection in a clonal seagrass. Nature Ecology \& Evolution 4:952- 


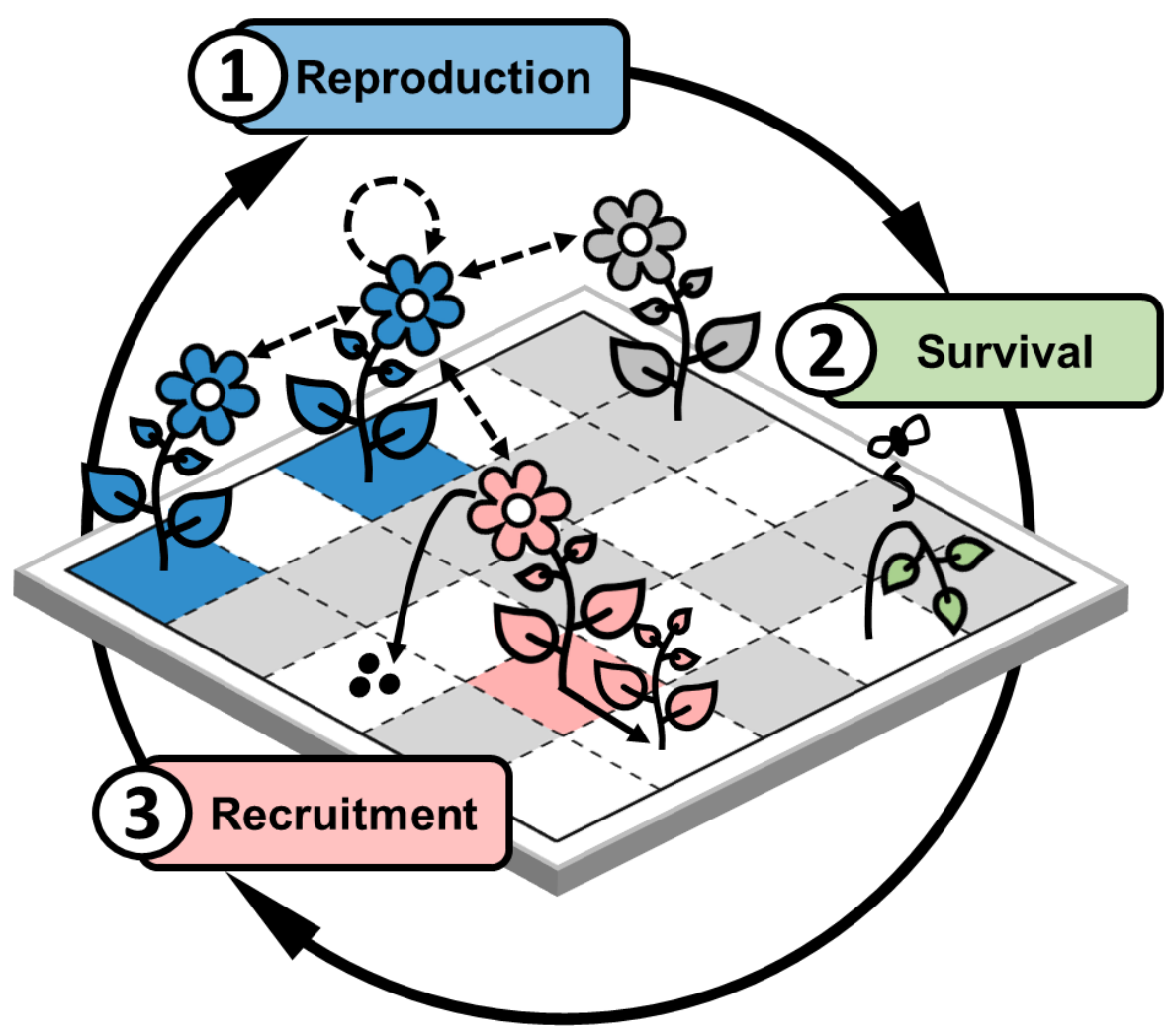

\begin{tabular}{|c|l|c|}
\hline Parameter & \multicolumn{1}{|c|}{ Description } & Values \\
\hline $\boldsymbol{D}$ & Lattice dimensions & 30 \\
\hline $\boldsymbol{N}$ & Population size & 900 \\
\hline $\boldsymbol{t}$ & Initial number of polyploids & 1 \\
\hline $\boldsymbol{o} \boldsymbol{v}$ & Ovule number & 5 \\
\hline $\boldsymbol{d}_{\boldsymbol{p}}$ & Average pollen dispersal & $0.5,2,5$ \\
\hline $\boldsymbol{u} \boldsymbol{g}$ & Unreduced gametes & $0-0.2$ \\
\hline $\boldsymbol{k}_{\mathbf{2}}$ & 2x selfed-seed inviability & $0.1,0.5,0.9$ \\
\hline $\boldsymbol{k}_{\mathbf{4}}$ & 4x selfed-seed inviability & $0.1,0.5,0.9$ \\
\hline $\boldsymbol{c}_{\boldsymbol{2}}$ & 2x per generation ramet production & $0,1,5$ \\
\hline $\boldsymbol{c}_{\boldsymbol{4}}$ & 4x per generation ramet production & $0,1,5$ \\
\hline $\boldsymbol{s}_{\boldsymbol{2}}$ & 2x per generation survival & $0-0.9$ \\
\hline $\boldsymbol{s}_{\boldsymbol{4}}$ & 4x per generation survival & $0-0.9$ \\
\hline $\boldsymbol{d}_{\boldsymbol{s}}$ & Average seed dispersal & $0.5,2,5$ \\
\hline $\boldsymbol{d}_{\boldsymbol{C 2}}$ & 2x average ramet dispersal & $0.5,5$ \\
\hline $\boldsymbol{d}_{\boldsymbol{C} \mathbf{4}}$ & 4x average ramet dispersal & $0.5,5$ \\
\hline
\end{tabular}

Figure 1: Simulation model workflow, and the adjustable parameters associated with each of the three main steps per generation. Models are initiated on a $D$ x $D$ population lattice containing both tetraploids ( $t$ individuals, $4 \mathrm{x}$, coloured cells) and diploids $(N-t$ individuals, 2x, grey cells), where each lattice cell can hold one individual. In Step 1, pollen is dispersed (dashed lines) within shoot, between shoots in the same genet (blue tetraploid genet), between members of the same cytotype (blue and pink tetraploids), and between cytotypes (blue tetraploid and grey diploid). Seeds are fertilized, and clonal ramets are produced. Individuals die in Step 2 according to their survival probability, leaving empty spaces in the population (white cells). In Step 3, empty cells are colonized by newly produced seeds or ramets. The surviving individuals and the new cohort of recruits then repeat this cycle, which continues for a 
A

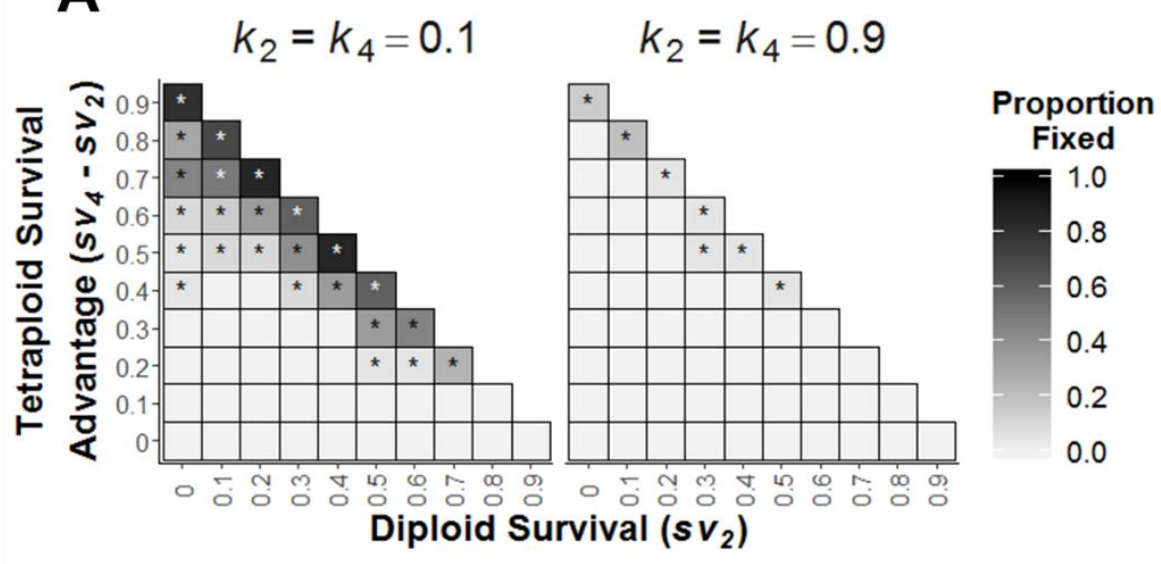

B

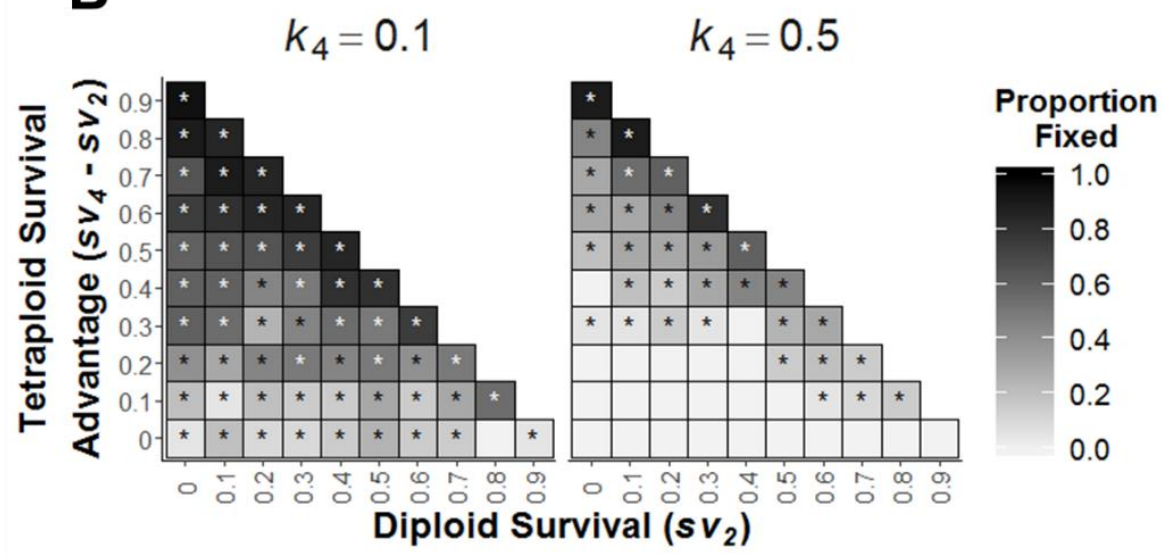

C
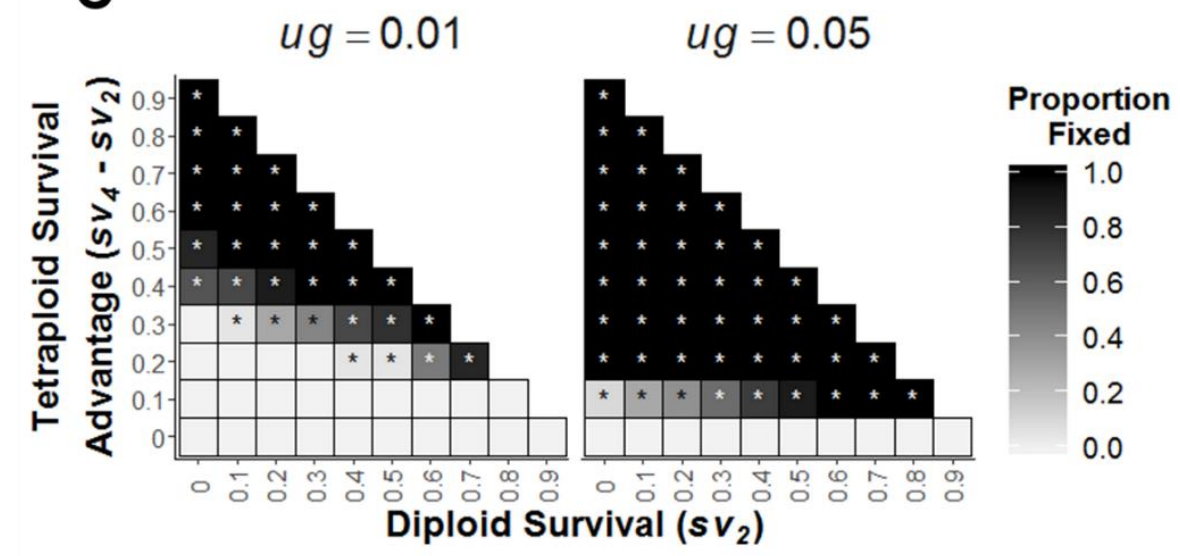

Figure 2: Effect of life history strategy on polyploid establishment for a range of diploid and tetraploid survival probabilities $\left(s v_{2}, s v_{4}\right)$. Establishment success is measured as the proportion of simulation replicates in which tetraploids spread to fixation, and exclude diploids from the population. Panel A shows establishment under equal selfed seed inviability $\left(k_{2}=k_{4}\right)$, while in Panel B tetraploid selfedseed inviability is lower than diploids ( $k_{2}=0.9, k_{4}$ value above panels). There is no unreduced gamete production in Panels A and B $(u g=0)$. In Panel C, unreduced gamete production is non-zero, and selfed-seed inviability is $k_{2}=k_{4}=0.1$. Asterisks denote parameter combinations where at least one 
A

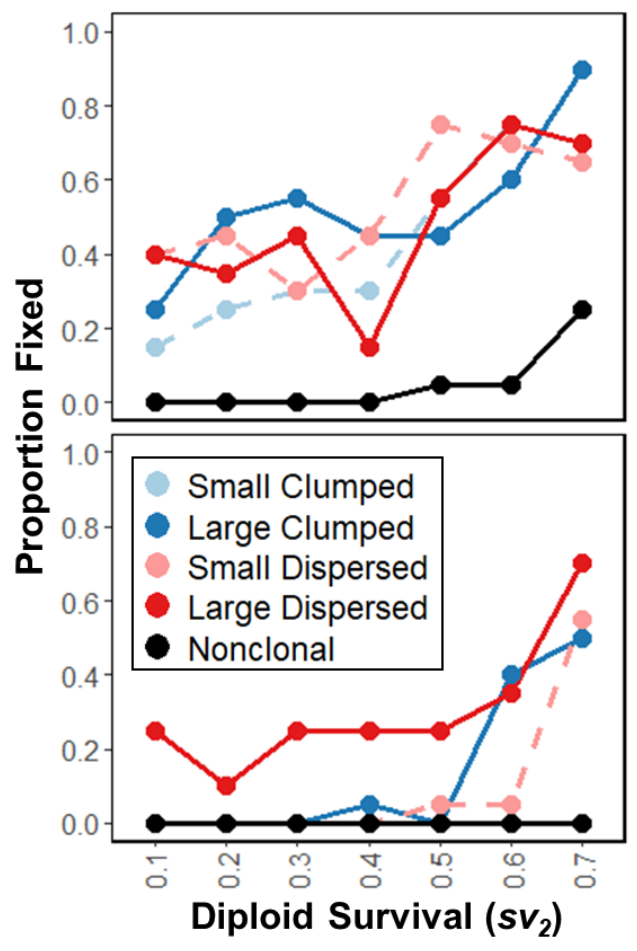

B
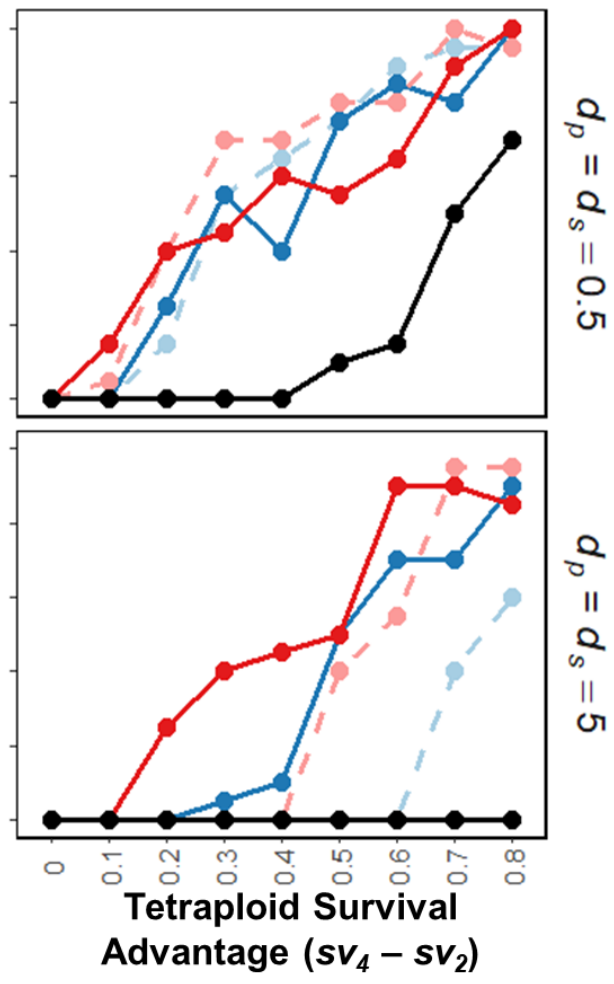

Figure 3: Influence of interactions between clonal strategy and life history on polyploid Dispersed $d_{C 2}=d_{C 4}=5$, red), and equal ramet production (genets are Small $c_{2}=c_{4}=1$, light dashed lines; or Large $c_{2}=c_{4}=5$, dark solid lines). In Panel A diploid survival probability $\left(s v_{2}\right)$ takes the values on the $\mathrm{x}$-axis and tetraploid survival $\left(s v_{4}\right)$ is 0.2 higher. In panel B diploid survival $\left(s v_{2}\right)$ is held at 0.1 , and tetraploids have a survival advantage $\left(s v_{4}-s v_{2}\right)$ according to the $\mathrm{x}$-axis. Average pollen $\left(d_{p}\right)$ and seed $\left(d_{s}\right)$ dispersal distances vary between rows in each panel. For all models, selfed-seed inviability is equal between cytotypes $\left(k_{2}=k_{4}=0.1\right)$, and there is no unreduced gamete production $(u g=0)$. 
971

972

973

974

975

976

977

978

979

980

981

982

983

984

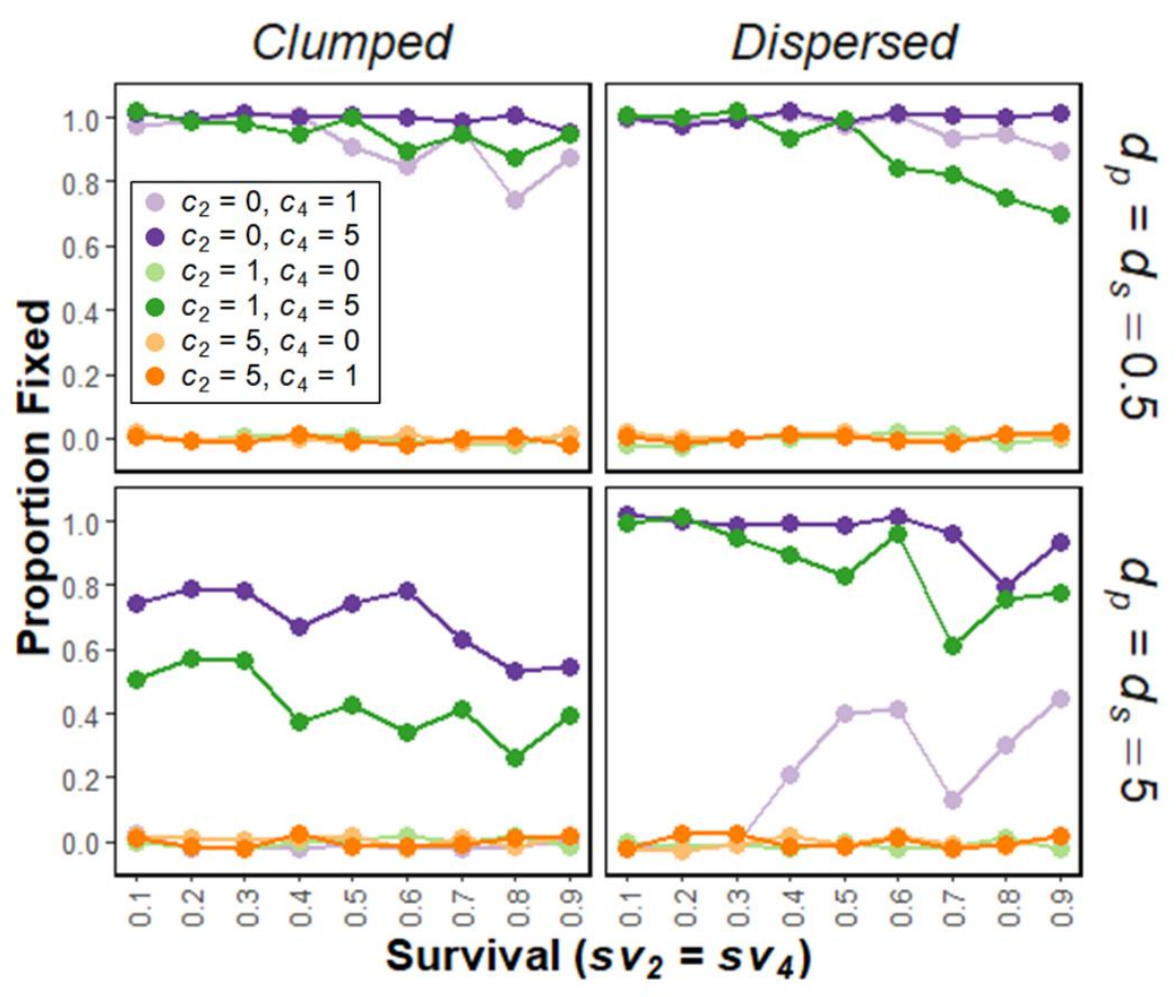

Figure 4: Polyploid establishment probability when diploids and tetraploids have unequal ramet production (i.e., unequal genet size, $c_{2} \neq c_{4}$ ). In comparison to diploids, tetraploids can produce a few more ramets $\left(c_{2}=0, c_{4}=1\right.$; light purple lines), many more ramets ( $c_{2}=0$ or $1, c_{4}=5$; dark purple and dark green lines), fewer ramets $\left(c_{2}=5, c_{4}=1\right.$; dark orange lines), or no ramets $\left(c_{2}=1\right.$ or $5, c_{4}=0$; light green and light orange lines). Clonal architecture is the same for both cytotypes, with the Clumped strategy in the left column $\left(d_{C 2}=d_{C 4}=0.5\right)$, and the Dispersed strategy in the right column $\left(d_{C 2}=d_{C 4}=\right.$ $5)$. Average pollen and seed dispersal distances $\left(d_{p}=d_{s}\right)$ varies between rows. Life history strategies are equal between cytotypes $\left(s v_{2}=s v_{4}\right)$, as shown on the $\mathrm{x}$-axis. For all models, selfed-seed inviability is equal between cytotypes $\left(k_{2}=k_{4}=0.1\right)$, and there is no unreduced gamete production $(u g=0)$. Points are slightly jittered vertically. 


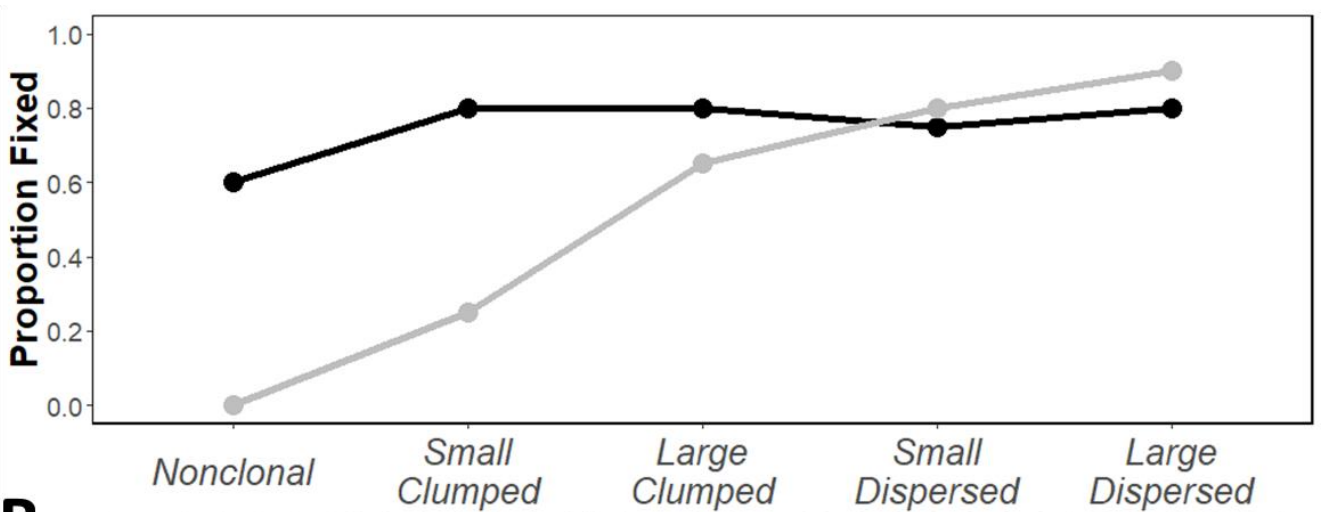

B

10
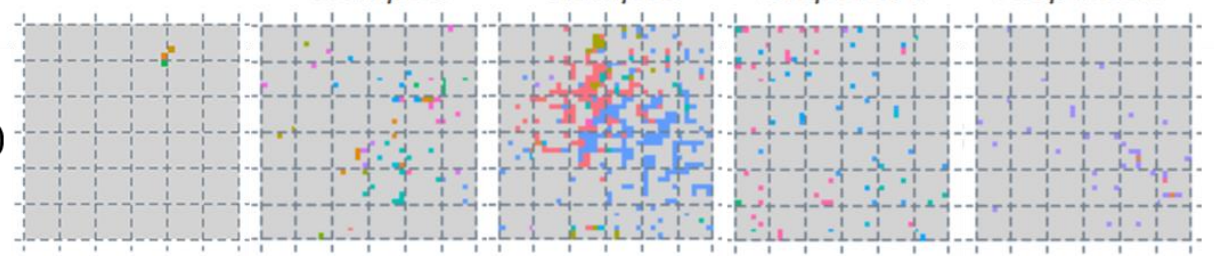

Figure 5: Contrasting the spread and establishment probability of nonclonal tetraploids and each clonal strategy. Panel A shows the proportion of simulations with successful polyploid establishment between nonclonal and clonal strategies (black $d_{p}=d_{s}=0.5$, grey $d_{p}=d_{s}=5$ ). Cytotypes have identical architecture (Clumped $d_{C 2}=d_{C 4}=0.5$; Dispersed $d_{C 2}=d_{C 4}=5$ ), and equal ramet production (genets are Small $c_{2}=c_{4}=1$; or Large $c_{2}=c_{4}=5$ ). In Panel B, tetraploids (coloured cells) expand into the 2dimensional population of diploids (grey cells), for $d_{p}=0.5$ (black line in A). Each colour represents a different tetraploid genet. For all models, $s v_{2}=0.5, s v_{4}=0.9$, selfed-seed inviability is equal between cytotypes $\left(k_{2}=k_{4}=0.1\right)$, and there is no unreduced gamete production $(u g=0)$. 
A
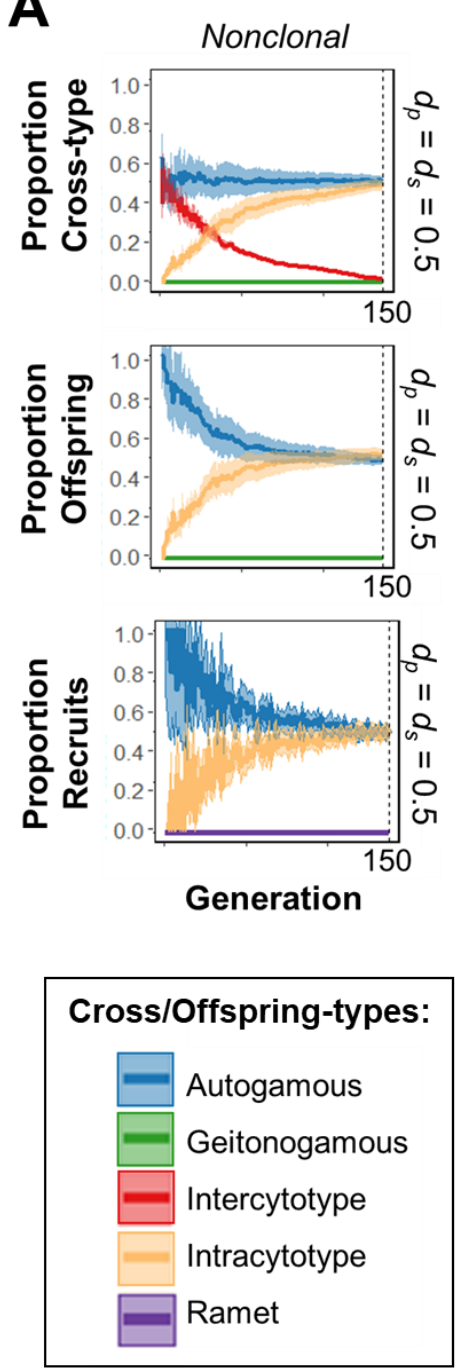
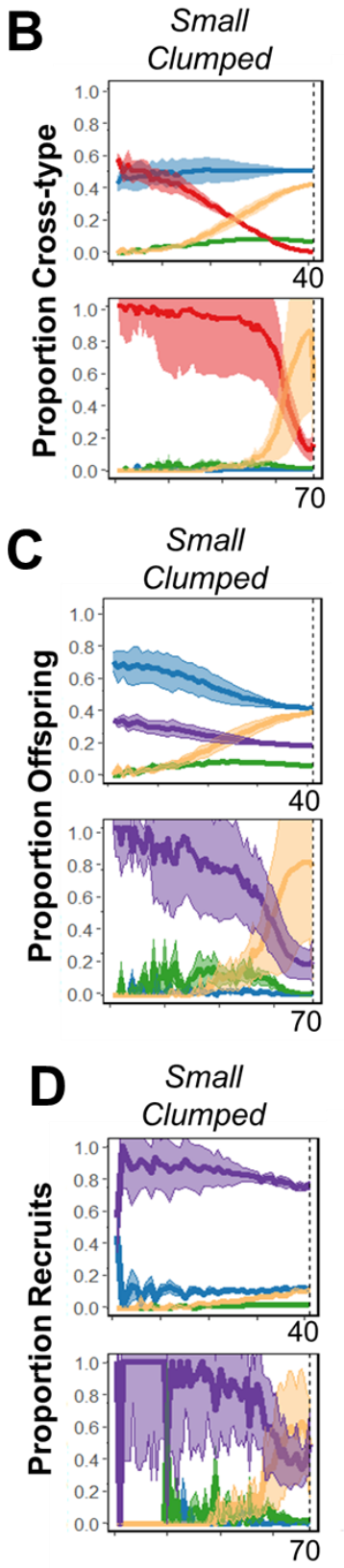

Large Clumped
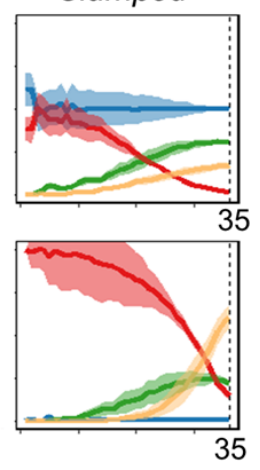

Large

Clumped

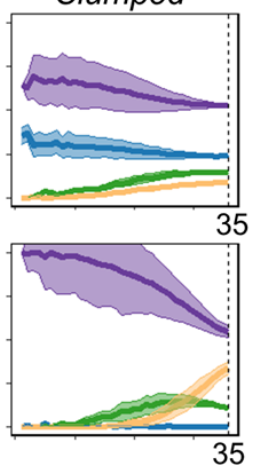

Large

Clumped
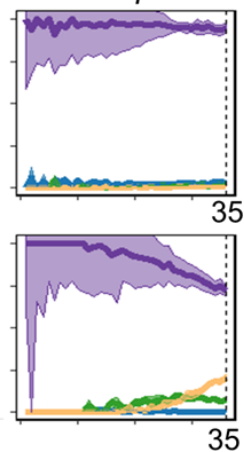

Small Dispersed
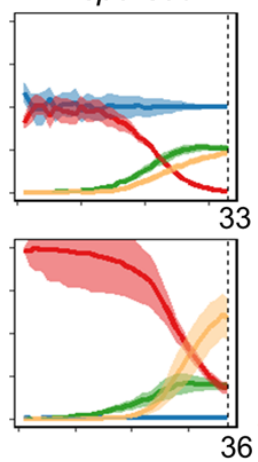

Small Dispersed

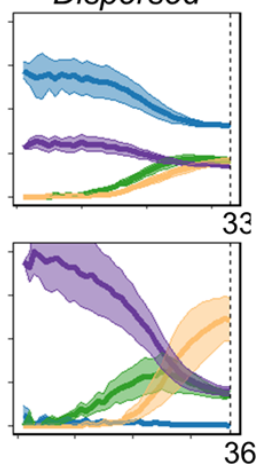

Small Dispersed
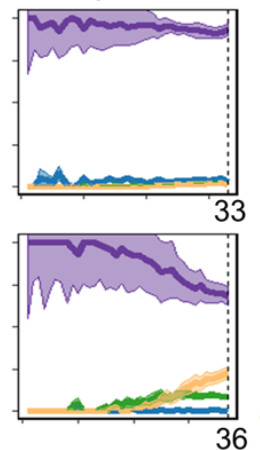

Large Dispersed
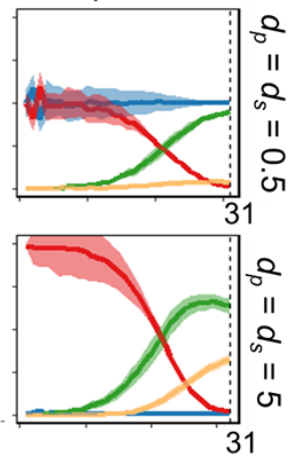

Large Dispersed

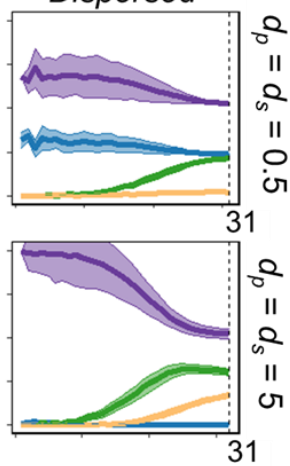

Large Dispersed
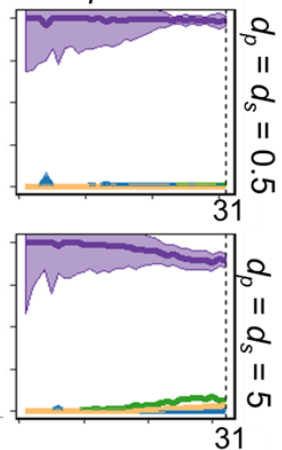

996

997

998

999

1000

1001

1002

1003

1004

1005

1006

1007

1008

1009

1010

Figure 6: Mating outcomes and offspring recruitment during polyploid establishment, averaged ( \pm SE) across simulation replicates per generation. Tetraploid mating events in Panel A (nonclonal) and Panel B (clonal) can result in four cross-types: autogamous self-fertilization (blue, cross-types \#16-17 in Table S1), geitonogamous self-fertilization (green, \#18-19), intercytotype mating (red, \#20), or intracytotype mating (yellow, \#22). The recruitment pool in Panel A (nonclonal) and Panel C (clonal) contains any viable sexual offspring and clonal ramets (purple) produced, which may then be recruited into the population as shown in Panel A (nonclonal) and Panel D (clonal). In Panel A all scenarios have $d_{p}=0.5$, as no establishment occurred when $d_{p}=5$. In Panels B - D, clonal strategy varies across columns, and average pollen and seed dispersal distance $\left(d_{p}=d_{s}\right)$ varies between rows. Cytotypes have identical architecture (Clumped $d_{C 2}=d_{C 4}=0.5$; Dispersed $d_{C 2}=d_{C 4}=5$ ), and equal ramet production (genets are Small $c_{2}=c_{4}=1$; or Large $c_{2}=c_{4}=5$ ). For all models, $s v_{2}=0.5, s v_{4}=0.9$, selfed-seed inviability is equal between cytotypes $\left(k_{2}=k_{4}=0.1\right)$, and there is no unreduced gamete production $(u g=0)$. Generations are truncated at the fastest tetraploid fixation time per parameter combination, indicated on the bottom right of each panel. 